\title{
Revisiting Minimal Lepton Flavour Violation in the light of leptonic CP violation
}

\author{
D.N. Dinh, ${ }^{a, b}$ L. Merlo, $^{c}$ S.T. Petcov ${ }^{d, e}$ and R. Vega-Álvarez ${ }^{c}$ \\ ${ }^{a}$ Mathematical and high energy physics group, Institute of physics, \\ Vietnam academy of science and technology, \\ 10 Dao Tan, Ba Dinh, Hanoi, Viet Nam \\ ${ }^{b}$ Department of Physics, University of Virginia, \\ Charlottesville, VA 22904-4714, U.S.A. \\ ${ }^{c}$ Departamento de Fúsica Teórica and Instituto de Fúsica Teórica, IFT-UAM/CSIC, \\ Universidad Autónoma de Madrid, \\ Cantoblanco, 28049, Madrid, Spain \\ ${ }^{d}$ SISSA and INFN-Sezione di Trieste, \\ Via Bonomea 265, 34136 Trieste, Italy \\ ${ }^{e}$ Kavli IPMU, University of Tokyo (WPI), \\ Tokyo, Japan \\ E-mail: dndinh@iop.vast.ac.vn, luca.merlo@uam.es, petcov@sissa.it, \\ roberto.vegaa@estudiante.uam.es
}

Abstract: The Minimal Lepton Flavour Violation (MLFV) framework is discussed after the recent indication for $\mathrm{CP}$ violation in the leptonic sector. Among the three distinct versions of MLFV, the one with degenerate right-handed neutrinos will be disfavoured, if this indication is confirmed. The predictions for leptonic radiative rare decays and muon conversion in nuclei are analysed, identifying strategies to disentangle the different MLFV scenarios. The claim that the present anomalies in the semi-leptonic $B$-meson decays can be explained within the MLFV context is critically re-examined concluding that such an explanation is not compatible with the present bounds from purely leptonic processes.

Keywords: CP violation, Effective Field Theories, Global Symmetries, Neutrino Physics ARXIV EPRINT: 1705.09284 


\section{Contents}

1 Introduction 1

2 Minimal (Lepton) Flavour Violation 4

2.1 The lepton sector 6

3 Phenomenology in the lepton sector $\quad 11$

$\begin{array}{lll}3.1 & \text { The LFV effective Lagrangian } & 12\end{array}$

$\begin{array}{lll}3.2 & \text { Rare radiative leptonic decays and conversion in nuclei } & 15\end{array}$

$\begin{array}{lll}3.2 .1 & \text { Bounds on the LFV scale } & 16\end{array}$

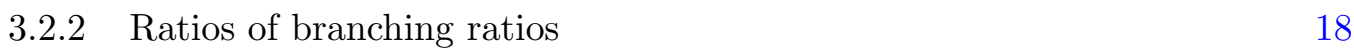

$4 \quad b \rightarrow s$ anomalies $\quad 23$

$4.1 \quad B$ semi-leptonic decays 24

5 Conclusions $\quad 25$

\section{Introduction}

The discovery [1-5] of a non-vanishing reactor angle $\theta_{13}^{\ell}$ in the lepton mixing matrix led to a huge fervour in the flavour community and to a deep catharsis in the model building approach.

When the value of this angle was still unknown, the closeness to a maximal mixing value of the atmospheric angle $\theta_{23}^{\ell}$ was suggesting a maximal oscillation between muonand tau-neutrinos: in terms of symmetries of the Lagrangian acting on the flavour space, it could be described by a discrete Abelian $Z_{2}$ symmetry, which, in turn, implied a vanishing reactor angle. The simplicity and the elegance of this pattern, i.e. one maximal angle and one vanishing one, convinced part of the community that Nature could have made us a favour and that neutrino physics could indeed be described, at least in the atmospheric and reactor sectors, by this texture $[6,7]$.

An approach followed for such constructions was to write a Lagrangian whose leading order terms described specific textures for the Yukawa matrices, leading to $\theta_{13}^{\ell}=0^{\circ}$ and $\theta_{23}^{\ell}=45^{\circ}$. Often, this was done such that the Yukawa matrix for the charged leptons was diagonal while the Yukawa matrix for the light active neutrinos was diagonalised by the so-called Tri-Bimaximal mixing matrix [8-10], which predicts, besides a vanishing reactor mixing angle and a maximal atmospheric one $\theta_{23}^{\ell}=45^{\circ}$, a solar angle satisfying to $\sin ^{2} \theta_{12}^{\ell}=1 / 3$, in a very good agreement with the neutrino oscillation data.

Pioneer models can be found in refs. [11-15], where the discrete non-Abelian group $A_{4}$ was taken as a flavour symmetry of the lepton sector. Several distinct proposals followed, 
i) attempting to achieve the Tri-Bimaximal pattern, but with other flavour symmetries (see for example refs. [16-19]); or ii) adopting other mixing patterns to describe neutrino oscillations, such as the Bimaximal mixing ${ }^{1}$ [21, 22], the Golden Ratio mixing [23, 24] and the Trimaximal mixing [25]; iii) analysing the possible perturbations or modifications to Bimaximal mixing, Tri-Bimaximal mixing etc., arising from the charged lepton sector [26-29], vi) implementing the so-called quark-lepton complementarity [30,31] which suggests that the lepton and quark sectors should not be treated independently, but a common dynamics could explain both the mixings [32-34]. Further details could be found for example in these reviews [35-40].

After the discovery of a non-vanishing $\theta_{13}^{\ell}$ and the improved sensitivity on the other two mixing angles, which pointed out that $\theta_{23}^{\ell}$ best fit is not $45^{\circ}$ (the most recent global fits on neutrino oscillation data can be found in refs. [41-43]), models based on discrete symmetries underwent to a deep rethinking. A few strategies have been suggested: introduction of additional parameters in preexisting minimal models, see for example refs. [44, 45]; implementation of features that allow sub-leading corrections only in specific directions in the flavour space [46-49]; search for alternative flavour symmetries or mixing patterns that lead already in first approximation to $\theta_{13}^{\ell} \neq 0^{\circ}$ and $\theta_{23}^{\ell} \neq 45^{\circ}[50,51]$. One can fairly say that the latest neutrino data can still be described in the context of discrete symmetries, but at the prize of fine-tunings and/or less minimal mechanisms.

Alternative approaches to discrete flavour model building strengthened after 2011 and, in particular, constructions based on continuous symmetries were considered interesting possibilities: models based on the simple U(1) (e.g. refs. [52-57]) or based on SU(3) (e.g. refs. $[58,59])$ or the so-called Minimal Flavour Violation (MFV) [60, 61], and its leptonic versions [62-65], dubbed MLFV. The latter is a setup where the flavour symmetry is identified with the symmetry of the fermionic kinetic terms, or in other words, the symmetry of the SM Lagrangian in the limit of vanishing Yukawa couplings: it is given by products of $\mathrm{U}(3)$ factors, one for each fermion spinor of the considered spectrum. Fermion masses and mixings are then described once the symmetry is broken. This approach allows to relate any source of flavour and CP violation in the SM and beyond to the Yukawa couplings, such that any flavour effect can be described in terms of fermion masses and mixing angles. The $\mathrm{M}(\mathrm{L}) \mathrm{FV}$ is not a complete model, as fermion masses and mixings are just described while their origin is not explained (attempts to improve with this respect can be found in refs. [66-75]). It is instead a framework where observed flavour violating observables are described in agreement with data and unobserved flavour violating signals are not expected to be observed with the current experimental sensitivities, but could be observable in the future planned experiments with significantly higher sensitivity, assuming the New Physics (NP) responsible for these phenomenology at the TeV scale or slightly higher [61-65, 76-89].

The recent indication of a relatively large Dirac CP violation in the lepton sector [41-43, 90-92] represented a new turning point in the sector. Present data prefer a non-zero Dirac CP phase, $\delta_{\mathrm{CP}}^{\ell}$, over CP conservation at more than $2 \sigma$ 's, depending on the

\footnotetext{
${ }^{1}$ Bimaximal mixing can be obtained by assuming the existence of an approximate $\mathrm{U}(1)$ symmetry corresponding to the conservation of the non-standard lepton charge $L^{\prime}=L_{e}-L_{\mu}-L_{\tau}$ and additional discrete $\mu-\tau$ symmetry [20].
} 
specific neutrino mass ordering. Moreover, the best fit value for the leptonic Jarlskog invariant, $J_{\mathrm{CP}}^{\ell} \simeq-0.033$ [42], is numerically much larger in magnitude than its quark sibling, $J_{\mathrm{CP}}^{\ell} \simeq 3.04 \times 10^{-5}$ [93], indicating potentially a much larger $\mathrm{CP}$ violation in the lepton sector than in the quark sector.

In the field of discrete flavour models, this indication translated into looking, for the first time, for approaches and/or contexts where, besides the mixing angles, also the lepton phase(s) were predicted: new models were presented with the CP symmetry as part of the full flavour symmetry [94-100]; studies on the mixing patterns and their modifications to provide realistic descriptions of oscillation data were performed [101-104]; an intense activity was dedicated to investigate sum rules involving neutrino masses, mixing angles and $\delta_{\mathrm{CP}}^{\ell}[102-109]$.

The indication for $\mathrm{CP}$ violation in the lepton sector also had an impact on models based on continuous flavour symmetries. In particular, one very popular version of MLFV [62] strictly requires $\mathrm{CP}$ conservation as a working assumption and therefore, if this indication is confirmed, this setup will be disfavoured.

The first goal of this paper is to update previous studies on MLFV in the light of the last global fit on neutrino oscillation data and to discuss the impact of the recent indication for CP violation in the lepton sector. Indeed, the last studies on MLFV date back to the original papers in 2005 [62,63] and 2011 [65], before the discovery of a non-vanishing $\theta_{13}^{\ell}$ and lacking any information about the leptonic CP phase.

The search for an explanation of the heterogeneity of fermion masses and mixings, the so-called Flavour Puzzle, is just a part of the Flavour Problem of particle physics. A second aspect of this problem is related to the fact that models involving NP typically introduce new sources of flavour violation. Identifying the mechanism which explains why the experimentally measured flavour violation is very much consistent with the SM predictions is a crucial aspect in flavour physics. The use of flavour symmetries turned out to be useful also with this respect: a very well-known example is the MFV setup, as previously discussed, whose construction was originally meant exactly to solve this aspect of the Flavour Problem. Promising results have been obtained also with smaller symmetries than the MFV ones, both continuous [110-115] and discrete [116-123].

The Flavour Problem becomes even more interesting after the indications for anomalies in the semi-leptonic $B$-meson decays: the angular observable $P_{5}^{\prime}$ in the $B \rightarrow K^{*} \mu^{+} \mu^{-}$decay presents a tension with the SM prediction of $3.7 \sigma$ [124, 125] and $2 \sigma$ [126], considering $\mathrm{LHCb}$ and Belle data, respectively; the Branching Ratio of $B_{s} \rightarrow \phi \mu^{+} \mu^{-}$is in tension with the SM prediction at $3.2 \sigma$ [127]; the ratio $R_{D^{*}}^{\ell} \equiv B R\left(\bar{B} \rightarrow D^{(*)} \tau \bar{\nu}\right)_{\exp } / B R\left(\bar{B} \rightarrow D^{(*)} \ell \bar{\nu}\right)_{\exp } \times$ $B R\left(\bar{B} \rightarrow D^{(*)} \ell \bar{\nu}\right)_{\mathrm{SM}} / B R\left(\bar{B} \rightarrow D^{(*)} \tau \bar{\nu}\right)_{\mathrm{SM}}$ with $\ell=e, \mu$ indicates a $3.9 \sigma$ violation of $\tau / \ell$ universality [128-132]; the ratio $R_{K} \equiv B R\left(B^{+} \rightarrow K^{+} \mu^{+} \mu^{-}\right) / B R\left(B^{+} \rightarrow K^{+} e^{+} e^{-}\right)$is in a $2.6 \sigma$ tension with the SM prediction [133], indicating lepton universality violation in the $e / \mu$ sector. The latter has been confirmed also by the recent announcement of the measure of $R_{K^{*}} \equiv B R\left(B^{0} \rightarrow K^{* 0} \mu^{+} \mu^{-}\right) / B R\left(B^{0} \rightarrow K^{* 0} e^{+} e^{-}\right)$is in a $2.4-2.5 \sigma(2.2-2.4 \sigma)$ tension with the SM prediction in the central- $q^{2}$ region (low- $q^{2}$ region) [134]. Under the assumption that these anomalies are due to NP, and not due to an underestimation of the hadronic effects [135-140] or due to a statistical fluctuation, a global analysis on $b \rightarrow s$ 
data can attempt to identify the properties of the underlying theory. Adopting an effective description, these results can be translated into constraints of the Wilson coefficients of the Hamiltonian describing $\Delta B=1$ decays: the results of such analysis [141-153] are that the anomalies can be explained with a modification of the Wilson coefficients $C_{9}$ and $C_{10}$ defined as

$$
\mathscr{H}_{\Delta B=1}^{\mathrm{eff}} \supset-\frac{4 G_{F}}{\sqrt{2}} \frac{e^{2}}{(4 \pi)^{2}} V_{t b} V_{t s}^{*}\left[\bar{s} \gamma_{\mu} P_{L} b\right]\left[\bar{\ell} \gamma^{\mu}\left(C_{9}+C_{10} \gamma_{5}\right) \ell\right]+\text { h.c. }
$$

where $V$ is the CKM matrix, $P_{L}=\left(1-\gamma_{5}\right) / 2$ is the usual left-handed (LH) chirality projector, $b$ and $s$ refer to the bottom and strange quarks, respectively, $\ell$ are the charged leptons, and the pre-factors refer to the traditional normalisation. Writing each of the coefficients as the sum of the purely SM contribution and the NP one, $C_{i}=C_{i}^{\mathrm{SM}}+\delta C_{i}$, the results of a one-operator-at-a-time analysis [151] suggest lepton universality violation in the $e / \mu$ sector quantifiable in

$$
\delta C_{9}^{e}=-\delta C_{10}^{e} \in[+0.56,+1.02] \quad \text { and } \quad \delta C_{9}^{\mu}=-\delta C_{10}^{\mu} \in[-0.81,-0.48] @ 1 \sigma,
$$

corresponding to $4.3 \sigma$ and $4.2 \sigma$ tension with the SM predictions, respectively.

The hypothetical underlying theory, which manifests itself at low energies with these features, will necessarily respect the SM gauge invariance, and therefore will also contribute to $b \rightarrow c$ processes and hopefully solve the $R_{D^{(*)}}^{\ell}$ anomalies.

Several attempts have been presented in the literature to explain the deficit on $C_{9}$ and/or $C_{10}$, including the MLFV approach: ref. [154] considers the version of MLFV introduced in ref. [62] and constraints on the Lagrangian parameters and on the Lepton Flavour Violating (LFV) scale have been obtained requiring to reproduce the values of $\delta C_{9}^{e}$ and $\delta C_{10}^{e}$ aforementioned.

A second goal of this paper is to revisit the results presented in ref. [154] considering the constraints from purely leptonic observables, such as radiative rare decays and $\mu \rightarrow e$ conversion in nuclei. Moreover, the analysis will be extended to the other versions of MLFV [65].

The structure of the paper can easily be deduced from the table of content: first, in section 2, basic concepts of MFV and MLFV will be recalled, underlying the differences between the distinct versions of MLFV; then, in section 3, several processes in the lepton sector will be discussed considering the last global fit on neutrino data and the recent indication for leptonic CP violation; in section 4 , the anomalies in the $b \rightarrow s$ decays will be discussed, pointing out the differences with respect to previous literature; finally, concluding remarks will be presented in section 5 .

\section{Minimal (Lepton) Flavour Violation}

If a theory of NP, with a characteristic scale of a few TeVs, behaves at low energy accordingly to the MFV ansatz, i.e. the SM Yukawa couplings are the only sources of flavour and $\mathrm{CP}$ violation even beyond the SM, then its flavour protection is guaranteed: the large majority of observed flavour processes in the quark sector are predicted in agreement with 
data [61, 76, 78-86, 155-160]; unseen flavour changing processes, for example leptonic radiative rare decays, are predicted to have strengths which are inside the present experimental sensitivity [62, 63, 65, 88, 161-164].

In the modern realisation of the MFV ansatz, the flavour symmetry corresponds to the one arising in the limit of vanishing Yukawa couplings. This massless Lagrangian is left invariant under a tridimensional unitary transformations in the flavour space associated to each fermion spinor. In the quark sector, it is given by

$$
\mathcal{G}_{Q} \times \mathrm{U}(1)_{B} \times \mathrm{U}(1)_{A^{u}} \times \mathrm{U}(1)_{A^{d}} \quad \text { with } \quad \mathcal{G}_{Q}=\mathrm{SU}(3)_{q_{L}} \times \mathrm{SU}(3)_{u_{R}} \times \mathrm{SU}(3)_{d_{R}},
$$

where $q_{L}$ refer to the $\mathrm{SU}(2)_{L}$-doublet of quarks, and $u_{R}$ and $d_{R}$ to the $\mathrm{SU}(2)_{L}$-singlets. The Abelian terms can be identified with the Baryon number, and with two axial rotations, in the up- and down-quark sectors respectively, which do not distinguish among the distinct families [165]. On the contrary, the non-Abelian factors rule the interactions among the generations and govern the amount of flavour violation: they are the key ingredients of $\mathrm{MFV}$ and will be in the focus of the analysis in which follows.

The explicit quark transformations read

$$
\begin{array}{lll}
q_{L} \sim(\mathbf{3}, 1,1)_{\mathcal{G}_{Q}} & u_{R} \sim(1, \mathbf{3}, 1)_{\mathcal{G}_{Q}} & d_{R} \sim(1,1, \mathbf{3})_{\mathcal{G}_{Q}} \\
q_{L} \rightarrow \mathcal{U}_{q_{L}} q_{L} & u_{R} \rightarrow \mathcal{U}_{u_{R}} u_{R} & d_{R} \rightarrow \mathcal{U}_{d_{R}} d_{R},
\end{array}
$$

where $\mathcal{U}_{i} \in \mathrm{SU}(3)_{i}$ are $3 \times 3$ unitary matrices acting in the flavour space. The quark Lagrangian is invariant under these transformations, except for the Yukawa interactions:

$$
\mathscr{L}_{Q}=-\bar{q}_{L} Y_{u} \tilde{H} u_{R}-\bar{q}_{L} Y_{d} H d_{R}+\text { h.c. },
$$

where $Y_{i}$ are $3 \times 3$ matrices in the flavour space, $H$ is the $\mathrm{SU}(2)_{L}$-double Higgs field, and $\tilde{H}=i \sigma_{2} H^{*}$. $\mathscr{L}_{Q}$ can be made invariant under $\mathcal{G}_{Q}$ promoting the Yukawa matrices to be spurion fields, i.e. auxiliary non-dynamical fields, denoted by $\mathcal{Y}_{u}$ and $\mathcal{Y}_{d}$, with specific transformation properties under the flavour symmetry:

$$
\begin{array}{ll}
\mathcal{Y}_{u} \sim(\mathbf{3}, \overline{\mathbf{3}}, 1)_{\mathcal{G}_{Q}} & \mathcal{Y}_{d} \sim(\mathbf{3}, 1, \overline{\mathbf{3}})_{\mathcal{G}_{Q}} \\
\mathcal{Y}_{u} \rightarrow \mathcal{U}_{q_{L}} \mathcal{Y}_{u} \mathcal{U}_{u_{R}}^{\dagger} & \mathcal{Y}_{d} \rightarrow \mathcal{U}_{q_{L}} \mathcal{Y}_{d} \mathcal{U}_{d_{R}}^{\dagger}
\end{array}
$$

Once the Yukawa spurions acquire a background value, the flavour symmetry is broken and in consequence fermions masses and mixings are generated. A useful choice for these background values is to identify them with the SM Yukawa couplings: in a given basis, $Y_{d}$ is diagonal and describes only down-type quark masses, while $Y_{u}$ contains non-diagonal entries and accounts for both up-type quark masses and the CKM matrix $V$ :

$$
\left\langle\mathcal{Y}_{u}\right\rangle \equiv Y_{u}=\frac{\sqrt{2}}{v} V^{\dagger} \hat{M}_{u}, \quad\left\langle\mathcal{Y}_{d}\right\rangle \equiv Y_{d}=\frac{\sqrt{2}}{v} \hat{M}_{d}
$$

where $v=246 \mathrm{GeV}$ is the Higgs vacuum expectation value (VEV) defined by $\left\langle H^{0}\right\rangle=v / \sqrt{2}$, and $\hat{M}_{u, d}$ are the diagonal mass matrices for up- and down-type quarks,

$$
\hat{M}_{u} \equiv \operatorname{diag}\left(m_{u}, m_{c}, m_{t}\right), \quad \hat{M}_{d} \equiv \operatorname{diag}\left(m_{d}, m_{s}, m_{b}\right) .
$$


When considering low-energy flavour processes, they can be described within the effective field theory approach through non-renormalisable operators suppressed by suitable powers of the scale associated to the messenger of the interaction. These structures could violate the flavour symmetry $\mathcal{G}_{Q}$, especially if they describe flavour changing observables. As for the Yukawa Lagrangian, a technical way out to recover flavour invariance is to insert powers of the Yukawa spurions. Once the spurions acquire background values, the corresponding processes are predicted in terms of quark masses and mixings. Several studies already appeared addressing this topic $[61,76,78-86,155-160]$ and, as already mentioned at the beginning of this section, the results show that flavour data in the quark sector are well described within the MFV(-like) approach. Indeed, the Yukawa spurions act as expanding parameters and processes described by effective operators with more insertions of the spurions obtain stronger suppressions. ${ }^{2}$

MFV, however, cannot be considered a complete flavour model, as there is not explanation of the origin of quark masses and mixings. There have been attempts to go from the effective-spurionic approach to a more fundamental description, promoting the Yukawa spurions to be dynamical fields, called flavons, acquiring a non-trivial VEV. The corresponding scalar potentials have been discussed extensively with interesting consequences [66-70]: a conclusive dynamical justification for quark masses and mixing is still lacking, but the results are encouraging as the potential minima lead, at leading order, to non-vanishing masses for top and bottom quarks and to no mixing.

\subsection{The lepton sector}

The lepton sector is more involved with respect to the quark one, due to the lack of knowledge on neutrino masses: indeed, while the charged lepton description mimics the one of down-quarks, light active neutrino masses, and then the lepton mixing, cannot be described within the SM.

Several ways out have been presented in the literature to provide a description for the lepton sector, and the focus here will be on two well-defined approaches, one maintaining the SM spectrum but relaxing the renormalisability criterium, and the other adding new particles in a still renormalisable theory.

Minimal Field Content (MFC). Giving up with renormalisability, active neutrino masses can be described via the so-called Weinberg operator [167], a non-renormalisable operator of canonical dimension 5 which breaks explicitly Lepton number by two units,

$$
\mathcal{O}_{W}=\frac{1}{2}\left(\overline{\ell_{L}^{c}} \tilde{H}^{*}\right) \frac{g_{\nu}}{\Lambda_{\mathrm{L}}}\left(\tilde{H}^{\dagger} \ell_{L}\right)
$$

where $\ell_{L}^{c} \equiv C{\overline{\ell_{L}}}^{T}, C$ being the charge conjugation matrix $\left(C^{-1} \gamma_{\mu} C=-\gamma_{\mu}^{T}\right), g_{\nu}$ is an adimensional symmetric $3 \times 3$ matrix in the flavour space and $\Lambda_{\mathrm{L}}$ is the scale of Lepton Number Violation (LNV). The flavour symmetry arising from the kinetic terms in this case is

$$
\mathcal{G}_{L} \times \mathrm{U}(1)_{L} \times \mathrm{U}(1)_{A^{e}} \quad \text { with } \quad \mathcal{G}_{L}=\mathrm{SU}(3)_{\ell_{L}} \times \mathrm{SU}(3)_{e_{R}},
$$

\footnotetext{
${ }^{2}$ The top Yukawa represents an exception as it cannot be technically taken as an expanding parameter. This aspect has been treated in refs. [166], where a resummation procedure has been illustrated.
} 
where $\mathrm{U}(1)_{L}$ is the Lepton number while $\mathrm{U}(1)_{A^{e}}$ is an axial rotation in $\ell_{L}$ and $e_{R}$, and the non-Abelian transformations of the leptons read

$$
\begin{array}{rlrl}
\ell_{L} & \sim(\mathbf{3}, 1)_{\mathcal{G}_{L}} & e_{R} \sim(1, \mathbf{3})_{\mathcal{G}_{L}} \\
\ell_{L} \rightarrow \mathcal{U}_{\ell_{L}} \ell_{L} & e_{R} \rightarrow \mathcal{U}_{e_{R}} e_{R} .
\end{array}
$$

The part of the Lagrangian describing lepton masses and mixings,

$$
\mathscr{L}_{L}=-\bar{\ell}_{L} Y_{e} H e_{R}-\mathcal{O}_{W}+\text { h.c. },
$$

is not invariant under $\mathcal{G}_{L}$, but this can be cured by promoting $Y_{e}$ and $g_{\nu}$ to be spurion fields, $\mathcal{Y}_{e}$ and $\mathscr{g}_{\nu}$, transforming as

$$
\begin{array}{ll}
\mathcal{Y}_{e} \sim(\mathbf{3}, \overline{\mathbf{3}})_{\mathcal{G}_{L}} & \mathscr{g}_{\nu} \sim(\overline{\mathbf{6}}, 1)_{\mathcal{G}_{L}} \\
\mathcal{Y}_{e} \rightarrow \mathcal{U}_{\ell_{L}} \mathcal{Y}_{e} \mathcal{U}_{e_{R}}^{\dagger} & \mathscr{g}_{\nu} \rightarrow \mathcal{U}_{\ell_{L} \mathscr{F}_{\nu}}^{*} \mathcal{U}_{\ell_{L}}^{\dagger} .
\end{array}
$$

Lepton masses and the PMNS matrix $U$ arise once $\mathcal{Y}_{e}$ and $\mathscr{g}_{\nu}$ acquire a background value that can be chosen to be

$$
\left\langle\mathcal{Y}_{e}\right\rangle \equiv Y_{e}=\frac{\sqrt{2}}{v} \hat{M}_{\ell}, \quad\left\langle g_{\nu}\right\rangle \equiv g_{\nu}=\frac{2 \Lambda_{\mathrm{L}}}{v^{2}} U^{*} \hat{M}_{\nu} U^{\dagger},
$$

with $\hat{M}_{\ell, \nu}$ being the diagonal matrices of the charged lepton and active neutrino mass eigenvalues,

$$
\hat{M}_{\ell} \equiv \operatorname{diag}\left(m_{e}, m_{\mu}, m_{\tau}\right), \quad \hat{M}_{\nu} \equiv \operatorname{diag}\left(m_{\nu_{1}}, m_{\nu_{2}}, m_{\nu_{3}}\right),
$$

and $U$ defined as the product of four matrices [93],

$$
U=R_{23}\left(\theta_{23}^{\ell}\right) \cdot R_{13}\left(\theta_{13}^{\ell}, \delta_{\mathrm{CP}}^{\ell}\right) \cdot R_{12}\left(\theta_{12}^{\ell}\right) \cdot \operatorname{diag}\left(1, e^{i \frac{\alpha_{21}}{2}}, e^{i \frac{\alpha_{31}}{2}}\right),
$$

with $R_{i j}\left(\theta_{i j}^{\ell}\right)$ a generic rotation of the angle $\theta_{i j}^{\ell}$ in the $i j$ sector, with the addition of the Dirac CP phase $\delta_{\mathrm{CP}}^{\ell}$ in the reactor sector, and $\alpha_{21,31}$ the Majorana phases [168].

As discussed for the quark case, $Y_{e}$ and $g_{\nu}$ act as expanding parameters: operators with more insertions of these spurions describe processes that receive stronger suppressions. This perturbative treatment requires, however, that the largest entries in $Y_{e}$ and $g_{\nu}$ are at most $\mathcal{O}(1)$. The charged lepton Yukawa satisfies to this condition as the largest entry is $\sim m_{\tau} / v$. The neutrino spurion $g_{\nu}$ is instead function of $\Lambda_{\mathrm{L}}$ : requiring that $\left|g_{\nu i j}\right|<1$ leads to an upper bound on the LNV scale, which depends on $\left|\left(U^{*} \hat{M}_{\nu} U^{\dagger}\right)_{i j}\right|$ that is a function of the type of neutrino mass spectrum ( $\mathrm{NO}$ or $\mathrm{IO}$ ), of the value of the lightest neutrino mass and of the values of the Majorana and Dirac CP violation phases. The lowest upper bound is given approximately by:

$$
\Lambda_{\mathrm{L}} \simeq \frac{v^{2}}{2} \frac{g_{\nu}}{\sqrt{\Delta m_{\mathrm{atm}}^{2}}} \lesssim 6 \times 10^{14} \mathrm{GeV} .
$$

It will be useful for the phenomenological discussion in the next sections to remember that the spurion combination $\mathscr{F}_{\nu}^{\dagger} \mathscr{\mathscr { g }} \nu$ transforms as $(\mathbf{8}, 1)_{\mathcal{G}_{L}}$ and to introduce the quantity

$$
\Delta \equiv g_{\nu}^{\dagger} g_{\nu}=\frac{4 \Lambda_{L}^{2}}{v^{4}} U \hat{M}_{\nu}^{2} U^{\dagger}
$$


Extended Field Content (EFC). Enlarging the SM spectrum by the addition of three RH neutrinos $N_{R}$ leads to the so-called type I Seesaw context [169-173], described by the following Lagrangian:

$$
\mathscr{L}_{\mathrm{L}-\mathrm{SS}}=-\bar{\ell}_{L} Y_{e} H e_{R}-\bar{\ell}_{L} Y_{\nu} \tilde{H} N_{R}-\frac{1}{2} \mu_{\mathrm{L}} \overline{N_{R}^{c}} Y_{N} N_{R}+\text { h.c. },
$$

where $Y_{e}, Y_{\nu}$ and $Y_{N}$ are adimensional $3 \times 3$ matrices in the flavour space, while $\mu_{\mathrm{L}}$ stands for the scale of Lepton number violation, broken by two units by the last term on the right of this equation. Assuming a hierarchy between $\mu_{\mathrm{L}}$ and $v, \mu_{\mathrm{L}} \gg v$, it is then possible to easily block-diagonalise the full $6 \times 6$ neutrino mass matrix, and obtain the induced masses for the light active neutrinos: in terms of the parameter $g_{\nu}$ appearing in the Weinberg operator in eq. (2.7), they are given by

$$
\frac{g_{\nu}^{\dagger}}{\Lambda_{\mathrm{L}}}=Y_{\nu} \frac{Y_{N}^{-1}}{\mu_{\mathrm{L}}} Y_{\nu}^{T}
$$

The fermionic kinetic terms of the SM extended with $3 \mathrm{RH}$ neutrinos manifest the following flavour symmetry:

$$
\mathcal{G}_{L} \times \mathrm{U}(1)_{L} \times \mathrm{U}(1)_{A^{e}} \times \mathrm{U}(1)_{A^{N}} \quad \text { with } \quad \mathcal{G}_{L}=\mathrm{SU}(3)_{\ell_{L}} \times \mathrm{SU}(3)_{e_{R}} \times \mathrm{SU}(3)_{N_{R}},
$$

under which leptons transform as

$$
\begin{aligned}
& \ell_{L} \sim(\mathbf{3}, 1,1)_{\mathcal{G}_{L}} \quad e_{R} \sim(1, \mathbf{3}, 1)_{\mathcal{G}_{L}} \quad N_{R} \sim(1,1, \mathbf{3})_{\mathcal{G}_{L}} \\
& \ell_{L} \rightarrow \mathcal{U}_{\ell_{L}} \ell_{L} \quad e_{R} \rightarrow \mathcal{U}_{e_{R}} e_{R} \quad N_{R} \rightarrow \mathcal{U}_{N_{R}} N_{R} \text {, }
\end{aligned}
$$

and where $\mathrm{U}(1)_{A^{N}}$ is an axial transformation associated to $N_{R}$ and $\mathrm{SU}(3)_{N_{R}}$ is a new rotation that mixes the three $\mathrm{RH}$ neutrinos. The Lagrangian in eq. (2.17) breaks explicitly $\mathcal{G}_{L}$ defined in eq. (2.19), but the invariance can be technically restored promoting $Y_{E}, Y_{\nu}$ and $Y_{N}$ to be spurions fields, $\mathcal{Y}_{E}, \mathcal{Y}_{\nu}$ and $\mathcal{Y}_{N}$, transforming as

$$
\begin{array}{lll}
\mathcal{Y}_{e} \sim(\mathbf{3}, \overline{\mathbf{3}}, 1)_{\mathcal{G}_{L}} & \mathcal{Y}_{\nu} \sim(\mathbf{3}, 1, \overline{\mathbf{3}})_{\mathcal{G}_{L}} & \mathcal{Y}_{N} \sim(1,1, \overline{\mathbf{6}})_{\mathcal{G}_{L}} \\
\mathcal{Y}_{e} \rightarrow \mathcal{U}_{\ell_{L}} \mathcal{Y}_{e} \mathcal{U}_{e_{R}}^{\dagger} & \mathcal{Y}_{\nu} \rightarrow \mathcal{U}_{\ell_{L}} \mathcal{Y}_{\nu} \mathcal{U}_{N_{R}}^{\dagger} & \mathcal{Y}_{N} \rightarrow \mathcal{U}_{N_{R}}^{*} \mathcal{Y}_{N} \mathcal{U}_{N_{R}}^{\dagger}
\end{array}
$$

Lepton masses and mixing are then described when these spurion fields acquire the following background values:

$$
\left\langle\mathcal{Y}_{e}\right\rangle \equiv Y_{e}=\frac{\sqrt{2}}{v} \hat{M}_{\ell}, \quad\left\langle\mathcal{Y}_{\nu}\right\rangle\left\langle\mathcal{Y}_{N}^{-1}\right\rangle\left\langle\mathcal{Y}_{\nu}^{T}\right\rangle \equiv Y_{\nu} Y_{N}^{-1} Y_{\nu}^{T}=\frac{2 \mu_{\mathrm{L}}}{v^{2}} U \hat{M}_{\nu} U^{T}
$$

Differently from the quark sector and the MFC lepton case, it is not possible to identify a unique choice for $\left\langle\mathcal{Y}_{\nu}\right\rangle$ and $\left\langle\mathcal{Y}_{N}\right\rangle$, as only the specific combination in eq. (2.22) can be associated to the neutrino mass eigenvalues and the PMNS matrix entries. This is a relevant aspect as it nullifies the MLFV flavour protection. Indeed, the basic building blocks for several processes, such as radiative leptonic decays or leptonic conversions, are fermionic bilinears of the type $\bar{\ell}_{L}^{i} \Gamma \ell_{L}^{j}, \bar{\ell}_{L}^{i} \Gamma \ell_{L}^{c j}, \bar{\ell}_{L}^{i} \Gamma e_{R}^{j}$ and $\bar{e}_{R}^{i} \Gamma e_{R}^{j}$, with $\Gamma$ standing for combination of Dirac $\gamma$ matrices and/or Pauli $\sigma$ matrices. In the unbroken phase, these terms are 
invariant under the flavour symmetry contracting the flavour indices with combinations of the spurions transforming as $(\mathbf{8}, 1,1)_{\mathcal{G}_{L}},(\mathbf{6}, 1,1)_{\mathcal{G}_{L}},(\mathbf{3}, \overline{\mathbf{3}}, 1)_{\mathcal{G}_{L}}$, and $(1, \mathbf{8}, 1)_{\mathcal{G}_{L}}$, among others. These spurion combinations are distinct from the combination of $\mathcal{Y}_{\nu}$ and $\mathcal{Y}_{N}$ t hat appears in eq. (2.22): a few examples are

$$
\begin{array}{ll}
(\mathbf{8}, 1,1)_{\mathcal{G}_{L}} & \mathcal{Y}_{\nu} \mathcal{Y}_{\nu}^{\dagger}, \mathcal{Y}_{e} \mathcal{Y}_{e}^{\dagger}, \mathcal{Y}_{\nu} \mathcal{Y}_{N}^{\dagger} \mathcal{Y}_{N} \mathcal{Y}_{\nu}^{\dagger},\left(\mathcal{Y}_{\nu} \mathcal{Y}_{\nu}^{\dagger}\right)^{2}, \ldots \\
(\mathbf{6}, 1,1)_{\mathcal{G}_{L}} & \mathcal{Y}_{\nu} \mathcal{Y}_{N}^{\dagger} \mathcal{Y}_{\nu}^{T}, \mathcal{Y}_{\nu} \mathcal{Y}_{N}^{\dagger} \mathcal{Y}_{N} \mathcal{Y}_{N}^{\dagger} \mathcal{Y}_{\nu}^{T}, \mathcal{Y}_{\nu} \mathcal{Y}_{N}^{\dagger} \mathcal{Y}_{\nu}^{T} \mathcal{Y}_{\nu}^{*} \mathcal{Y}_{\nu}^{T}, \ldots \\
(\mathbf{3}, \overline{\mathbf{3}}, 1)_{\mathcal{G}_{L}} & \mathcal{Y}_{e}, \mathcal{Y}_{\nu} \mathcal{Y}_{\nu}^{\dagger} \mathcal{Y}_{e}, \mathcal{Y}_{e} \mathcal{Y}_{e}^{\dagger} \mathcal{Y}_{e}, \mathcal{Y}_{\nu} \mathcal{Y}_{N}^{\dagger} \mathcal{Y}_{N} \mathcal{Y}_{\nu}^{\dagger} \mathcal{Y}_{e}, \ldots \\
(1, \mathbf{8}, 1)_{\mathcal{G}_{L}} & \mathcal{Y}_{e}^{\dagger} \mathcal{Y}_{e}, \mathcal{Y}_{e}^{\dagger} \mathcal{Y}_{\nu} \mathcal{Y}_{\nu}^{\dagger} \mathcal{Y}_{e}, \mathcal{Y}_{e}^{\dagger} \mathcal{Y}_{\nu} \mathcal{Y}_{N}^{\dagger} \mathcal{Y}_{N} \mathcal{Y}_{\nu}^{\dagger} \mathcal{Y}_{e}, \ldots
\end{array}
$$

In consequence, one concludes that it is not possible to express any flavour changing process involving leptons in terms of lepton masses and mixings, losing in this way the predictive power of MLFV.

This problem can be solved, and predictivity can be recovered, if all the information of neutrino masses and mixing would be encoded into only one spurion background among $Y_{\nu}$ and $Y_{N}$, being the other proportional to the identity matrix. Technically, this corresponds to break $\mathcal{G}_{L}$ following two natural criteria.

$\mathrm{I}): \mathcal{G}_{L} \rightarrow \mathrm{SU}(3)_{\ell_{L}} \times \mathrm{SU}(3)_{e_{R}} \times \mathrm{SO}(3)_{N_{R}} \times C P[62,63]$.

Under the assumption that the three RH neutrinos are degenerate in mass, i.e. $Y_{N} \propto \mathbb{1}$, $\mathrm{SO}(3)_{N_{R}}$ is broken down to $\mathrm{SO}(3)_{N_{R}}$ and the transformation $\mathcal{U}_{N_{R}}$ in eq. (2.20) is then an orthogonal matrix. The additional assumption of no $\mathrm{CP}$ violation in the lepton sector is meant to force $Y_{e}$ and $Y_{\nu}$ to be real. ${ }^{3}$ With this simplifications, all flavour changing effects involving leptons can be written in terms of $Y_{\nu} Y_{\nu}^{T}$ and $Y_{e}$, as can be easily deduced from eq. (2.23). In this case, eq. (2.22) simplifies to

$$
Y_{\nu} Y_{\nu}^{T}=\frac{2 \mu_{\mathrm{L}}}{v^{2}} U \hat{M}_{\nu} U^{T} \equiv \Delta
$$

eventually redefining $\mu_{\mathrm{L}}$ by reabsorbing the norm of $Y_{N}$, and therefore any flavour changing process can be described in terms of lepton masses and mixings. The last equivalence in the previous equation is a definition that will be useful in the phenomenological analysis.

As for the MFC case, requiring that the spurions respect the perturbativity regime leads to an upper bound on the LNV scale:

$$
\mu_{\mathrm{L}} \simeq \frac{v^{2}}{2} \frac{Y_{\nu} Y_{\nu}^{T}}{\sqrt{\Delta m_{\mathrm{atm}}^{2}}} \lesssim 6 \times 10^{14} \mathrm{GeV},
$$

numerically the same as the one in eq. (2.15).

\footnotetext{
${ }^{3}$ Strictly speaking, the condition of CP conservation in the leptonic sector forces the Dirac CP phase to be equal to $\delta_{\mathrm{CP}}^{\ell}=\{0, \pi\}$ and the Majorana CP phases to be $\alpha_{21,31}=\{0, \pi, 2 \pi\}$. However, $Y_{\nu}$ is real only if $\alpha_{21,31}=\{0,2 \pi\}$, and therefore $\alpha_{21,31}=\pi$ needs to be disregarded in order to guarantee predictivity. The CP conservation condition assumed in this context is then stronger than the strict definition.
} 
II): $\mathcal{G}_{L} \rightarrow \mathrm{SU}(3)_{\ell_{L}+N_{R}} \times \mathrm{SU}(3)_{e_{R}}$ [65].

Assuming that the three $\mathrm{RH}$ neutrinos transform as a triplet under the same symmetry group of the lepton doublets,

$$
\ell_{L}, N_{R} \sim(\mathbf{3}, 1)_{\mathcal{G}_{L}} \quad e_{R} \sim(1, \mathbf{3})_{\mathcal{G}_{L}},
$$

then the Schur's Lemma guarantees that $\mathcal{Y}_{\nu}$ transforms as a singlet of the symmetry group and then $Y_{\nu}$ is a unitary matrix $[174,175]$, which can always be rotated to the identity matrix by a suitable unitary transformation acting only on the RH neutrinos. The only sensible quantities in this context are $\mathcal{Y}_{e}$ and $\mathcal{Y}_{N}$, which now transform as

$$
\mathcal{Y}_{e} \sim(\mathbf{3}, \overline{\mathbf{3}})_{\mathcal{G}_{L}} \quad \mathcal{Y}_{N} \sim(\overline{\mathbf{6}}, 1)_{\mathcal{G}_{L}} .
$$

The background value of $\mathcal{Y}_{N}$ would eventually encode the norm of $Y_{\nu}$, in order to consistently take $Y_{\nu}=\mathbb{1}$. In this basis, neutrino masses and the lepton mixing are encoded uniquely into $Y_{N}$,

$$
Y_{N}=\frac{v^{2}}{2 \mu_{\mathrm{L}}} U^{*} \hat{M}_{\nu}^{-1} U^{\dagger} .
$$

Moreover, all the spurion combinations in eq. (2.23) can be written only in terms of $Y_{N}$ and $Y_{e}$ and therefore any flavour changing process can be predicted in terms of lepton masses and mixing. It will be useful in the phenomenological analysis that follows to introduce the quantity

$$
\Delta \equiv Y_{N}^{\dagger} Y_{N}=\frac{v^{4}}{4 \mu_{L}^{2}} U \hat{M}_{\nu}^{-2} U^{\dagger} .
$$

Contrary to what occurs in the MFC and the EFCI cases, the perturbativity condition on $Y_{N}$ allows to extract a lower bound on the LNV scale:

$$
\mu_{\mathrm{L}} \simeq \frac{v^{2}}{2} \frac{Y_{N}^{-1}}{\sqrt{\Delta m_{\mathrm{atm}}^{2}}} \gtrsim 6 \times 10^{14} \mathrm{GeV} .
$$

Similarly to what discussed for the quark sector, none of the two versions of the MLFV provide an explanation for the origin of lepton masses and mixing, and therefore cannot be considered complete models. In refs. [72-74] attempts have been presented to provide a dynamical explanation for the flavour puzzle in the lepton sector: as for the quark sector, the results are not conclusive, but highlighted interesting features. Indeed, for the MLFV version with an $\mathrm{SO}(3)_{N_{R}}$ symmetry factor associated to the $\mathrm{RH}$ neutrinos, the minima of the scalar potential, constructed by promoting $\mathcal{Y}_{e}$ and $\mathcal{Y}_{\nu}$ to be dynamical fields, allow a maximal mixing and a relative maximal Majorana $\mathrm{CP}$ phase between two almost degenerate neutrino mass eigenvalues. This seems to suggest that the large angles in the lepton sector could be due to the Majorana nature of neutrinos, in contrast with the quark sector where this does not occur.

No dedicated analysis of the scalar potential arising in the second version of MLFV has appeared in the literature, although the results are not expected to be much different from the ones in the quark sector. However, as a conclusive mechanism to explain lepton masses and mixing is still lacking, both the versions of MLFV remain valid possibilities. 


\begin{tabular}{|l|c|c|}
\hline & Normal Ordering & Inverted Ordering \\
\hline $\sin ^{2} \theta_{12}^{\ell}$ & $0.306 \pm 0.012$ & $0.306 \pm 0.012$ \\
$\sin ^{2} \theta_{23}^{\ell}$ & $0.441_{-0.021}^{+0.027}$ & $0.587_{-0.024}^{+0.020}$ \\
$\sin ^{2} \theta_{13}^{\ell}$ & $0.02166 \pm 0.00075$ & $0.02179 \pm 0.00076$ \\
$\delta_{\mathrm{CP}}^{\ell}{ }^{\circ}$ & $261_{-59}^{+51}$ & $277_{-46}^{+40}$ \\
$\Delta m_{\mathrm{sol}}^{2} / 10^{-5} \mathrm{eV}^{2}$ & $7.50_{-0.17}^{+0.19}$ & $7.50_{-0.17}^{+0.19}$ \\
$\Delta m_{\mathrm{atm}}^{2} / 10^{-3} \mathrm{eV}^{2}$ & $2.524_{-0.040}^{+0.039}$ & $2.514_{-0.041}^{+0.0 .38}$ \\
\hline
\end{tabular}

Table 1. Three-flavour oscillation parameters from the global fit in ref. [42]. The results in the second and third columns refer to the Normal and the Inverted Orderings, respectively. The notation has been chosen such that $\Delta m_{\text {sol }}^{2} \equiv m_{\nu_{2}}^{2}-m_{\nu_{1}}^{2}$, and $\Delta m_{\text {atm }}^{2} \equiv m_{\nu_{3}}^{2}-m_{\nu_{1}}^{2}$ for NO and $\Delta m_{\mathrm{atm}}^{2} \equiv m_{\nu_{2}}^{2}-m_{\nu_{3}}^{2}$ for IO. The errors reported correspond to the $1 \sigma$ uncertainties.

As anticipated in section 1, the recent indication for a relatively large leptonic CP violation, if confirmed, would disfavour EFCI, due to the required reality of $Y_{\nu}$. However, in the present discussion and in the analysis that follows, EFCI will not be discarded yet, as the assumption of $\mathrm{CP}$ conservation is a distinctive feature of this low-energy description of the lepton sector, but could be avoided in more fundamental ones. Indeed, a model constructed upon the gauged lepton flavour symmetry $\mathrm{SU}(3)_{\ell_{L}} \times \mathrm{SU}(3)_{e_{R}} \times \mathrm{SO}(3)_{N_{R}}$, without any further hypothesis on $\mathrm{CP}$ in the lepton sector, is shown in ref. [88] to be as predictive as EFCI: indeed, with the Dirac CP phase taken at its best fit value, this gauged flavour model presents several phenomenological results similar to the ones of EFCI discussed in refs. [62, 63]. This motivates to consider EFCI as a valid context to describe lepton flavour observables, even if results which show a strong dependence on the value of the Dirac CP phase should be taken with a grain of salt.

\section{Phenomenology in the lepton sector}

In this section, the phenomenology associated to the MFC, EFCI and EFCII cases will be discussed considering specifically leptonic radiative rare decays and $\mu \rightarrow e$ conversion in nuclei. While these analyses have already been presented in the original MLFV papers $[62,63,65]$, in the review part of the present paper the latest discovered value of the reactor angle and the recent indication of non-vanishing $\mathrm{CP}$ phase in the leptonic sector will be considered.

The input data that will be used in what follows are the PDG values for the charged lepton masses [93]

$$
m_{e}=0.51 \mathrm{MeV}, \quad m_{\mu}=105.66 \mathrm{MeV}, \quad m_{\tau}=1776.86 \pm 0.12 \mathrm{MeV},
$$

where the electron and muon masses are taken without errors as the sensitivities are negligible, and the results of the neutrino oscillation fit from ref. [42] reported in table 1.

The value of the lightest neutrino mass and the neutrino mass ordering are still unknown. For this reason, the results of this section will be discussed in terms of the values 
of the lightest neutrino mass and for both the Normal Ordering (NO) and the Inverted Ordering (IO). The measured parameters are taken considering their $2 \sigma$ error bands: ${ }^{4}$ this is to underly the impact of the raising indication for a leptonic $\mathrm{CP}$ violation.

\subsection{The LFV effective Lagrangian}

The rates of charged LFV processes, i.e. $\mu \rightarrow e+\gamma, \mu \rightarrow 3 e$, and $\mu \rightarrow e$ conversion in nuclei among others, are predicted to be unobservably small in the minimal extension of the SM with light massive Dirac neutrinos, in which the total lepton charge is conserved [180]. As a consequence, the rates of such processes have a remarkable sensitivity to NP contributions.

The main observables that will be discussed here are lepton radiative rare decays and $\mu \rightarrow e$ conversion in nuclei. Other leptonic observables which are typically very sensible to NP are $\ell \rightarrow \ell^{\prime} \ell^{\prime} \ell^{\prime \prime}$ decays, and especially the $\mu \rightarrow 3 e$ decay, given the significant increase of the sensitivity of the planned experiments. However, these processes do not provide additional information for the results that will be obtained in the following, and therefore they will not be further considered.

Assuming the presence of new physics at the scale $\Lambda_{\mathrm{LFV}}$ responsible for these observables characterised by a much lower typical energy, one can adopt the description in terms of an effective Lagrangian: ${ }^{5}$ the relevant terms are then given $b^{6}$

$$
\mathscr{L}_{\mathrm{LFV}}^{\mathrm{eff}}=\frac{1}{\Lambda_{\mathrm{LFV}}^{2}} \sum_{i=1}^{5} c_{L L}^{(i)} \mathcal{O}_{L L}^{(i)}+\frac{1}{\Lambda_{\mathrm{LFV}}^{2}}\left(\sum_{j=1}^{2} c_{R L}^{(j)} \mathcal{O}_{R L}^{(j)}+\text { h.c. }\right),
$$

where the Lagrangian parameters are real coefficients ${ }^{7}$ of order 1 and the operators have the form: ${ }^{8}$

$$
\begin{aligned}
\mathcal{O}_{L L}^{(1)} & =i \bar{\ell} \gamma^{\mu} \ell_{L} H^{\dagger} D_{\mu} H, & \mathcal{O}_{L L}^{(2)} & =i \bar{\ell} \gamma^{\mu} \sigma^{a} \ell_{L} H^{\dagger} \sigma^{a} D_{\mu} H, \\
\mathcal{O}_{L L}^{(3)} & =\bar{\ell} \gamma^{\mu} \ell_{L} \bar{q} \gamma_{\mu} q_{L}, & \mathcal{O}_{L L}^{(4 d)} & =\bar{\ell} \gamma^{\mu} \ell_{L} \bar{d} \gamma_{\mu} d_{R}, \\
\mathcal{O}_{L L}^{(4 u)} & =\bar{\ell} \gamma^{\mu} \ell_{L} \bar{u} \gamma_{\mu} u_{R}, & \mathcal{O}_{L L}^{(5)} & =\bar{\ell} \gamma^{\mu} \sigma^{a} \ell_{L} \bar{q} \gamma_{\mu} \sigma^{a} q_{L}, \\
\mathcal{O}_{R L}^{(1)} & =g^{\prime} \bar{\ell} H \sigma^{\mu \nu} e_{R} B_{\mu \nu}, & \mathcal{O}_{R L}^{(2)} & =g \bar{\ell} H \sigma^{\mu \nu} \sigma^{a} e_{R} W_{\mu \nu}^{a} .
\end{aligned}
$$

\footnotetext{
${ }^{4}$ EW running effects [176-179] are negligible in the analysis presented here.

${ }^{5}$ The effective Lagrangian reported here corresponds to the linearly realised EWSB. An alternative would be to considered a non-linear realisation and the corresponding effective Lagrangian dubbed HEFT [181-186]. In this context, however, a much larger number of operators should be taken into consideration and a slightly different phenomenology is expected [187-196]. The focus in this paper is on the linear EWSB realisation and therefore the HEFT Lagrangian will not be considered in what follows.

${ }^{6} \mathrm{~A}$ few other operators are usually considered in the effective Lagrangian associated to these LFV observables, but the corresponding effects are negligible. See ref. [62] for further details.

${ }^{7}$ The reality of the Lagrangian parameters guarantees that no sources of $\mathrm{CP}$ violation are introduced beyond the SM. A justification of this approach can be found in ref. [78].

${ }^{8}$ The notation chosen for the effective operators matches the one of the original MLFV paper [62]. It is nowadays common to adopt an other operator basis introduced in refs. [197, 198]. The link between the two bases is given by:

$$
\mathcal{O}_{L L}^{(1)} \rightarrow Q_{\varphi \ell}^{(1)}, \quad \mathcal{O}_{L L}^{(2)} \rightarrow Q_{\varphi \ell}^{(3)}, \quad \mathcal{O}_{L L}^{(3)} \rightarrow Q_{\ell q}^{(1)}, \quad \mathcal{O}_{L L}^{(4 d)} \rightarrow Q_{\ell d},
$$$$
\mathcal{O}_{L L}^{(4 u)} \rightarrow Q_{\ell d}, \quad \mathcal{O}_{L L}^{(5)} \rightarrow Q_{\ell q}^{(3)}, \quad \mathcal{O}_{R L}^{(1)} \rightarrow Q_{e B}, \quad \mathcal{O}_{R L}^{(2)} \rightarrow Q_{e W} .
$$ 
The $\mathcal{O}_{L L}^{(i)}$ structures are invariant under the flavour symmetries without the necessity of introducing any spurion field, but they can only contribute to flavour conserving observables. The LFV processes aforementioned can only be described by the insertion of specific spurion combinations transforming as $\mathbf{8}$ under $\mathrm{SU}(3)_{\ell_{L}}$, whose flavour indices are contracted with those of the lepton bilinear $\bar{\ell}_{L}^{i} \Gamma \ell_{L}^{j}$ in $\mathcal{O}_{L L}^{(i)}, \Gamma$ being a suitable combination of Dirac and/or Pauli matrices. The specific spurion combinations depend on the considered model: some examples are $\mathscr{g}_{\nu}^{\dagger} \mathscr{g}_{\nu}$ in MFC, $\mathcal{Y}_{\nu} \mathcal{Y}_{\nu}^{\dagger}$ in EFCI and $\mathcal{Y}_{\nu} \mathcal{Y}_{N}^{\dagger} \mathcal{Y}_{N} \mathcal{Y}_{\nu}^{\dagger}$ in EFCII. Interestingly, once the spurions acquire their background values, these combinations reduce to the expressions for $\Delta$ in eqs. (2.16), (2.24) and (2.29), respectively.

The $\mathcal{O}_{R L}^{(i)}$ operators, instead, are not invariant under the flavour symmetry $\mathcal{G}_{L}$ and require the insertion of spurion combinations transforming as $(\mathbf{3}, \overline{\mathbf{3}})$ under $\mathrm{SU}(3)_{\ell_{L}} \times \mathrm{SU}(3)_{e_{R}}$. The simplest combination of this kind is the charged lepton Yukawa spurion $\mathcal{Y}_{e}$, whose background value, however, is diagonal. Requiring as well that these structures describe LFV processes, it is necessary to insert more elaborated combinations: some examples are $\mathscr{g}_{\nu}^{\dagger} \mathscr{q}_{\nu} \mathcal{Y}_{e}$ in MFC, $\mathcal{Y}_{\nu} \mathcal{Y}_{\nu}^{\dagger} \mathcal{Y}_{e}$ in EFCI and $\mathcal{Y}_{N}^{\dagger} \mathcal{Y}_{N} \mathcal{Y}_{e}$ in EFCII. Once the spurions acquire background values, these combinations reduce to $\Delta Y_{e}$, with the specific expression for $\Delta$ depending on the case considered.

From the previous discussion one can deduce that the relevant quantity that allows to describe LFV processes in terms of lepton masses and mixings is $\Delta$, beside the diagonal matrix $Y_{e}$. It is then instructive to explicitly write the expression for $\Delta$ in the three cases under consideration and distinguishing between the NO and the IO for the neutrino mass spectrum. ${ }^{9}$

1. Minimal Field Content $\mathcal{G}_{L}=\mathrm{SU}(3)_{\ell_{L}} \times \mathrm{SU}(3)_{e_{R}}$. Expliciting eq. (2.16), the offdiagonal entries of $\Delta$ can be written as

$$
\begin{aligned}
& \Delta_{\mu e}=\frac{4 \Lambda_{\mathrm{L}}^{2}}{v^{4}}\left.s_{12} c_{12} c_{23} c_{13}\left(m_{\nu_{B}}-m_{\nu_{A}}\right)+s_{23} s_{13} c_{13} e^{i \delta}\left(m_{\nu_{C}}-s_{12}^{2} m_{\nu_{B}}-c_{12}^{2} m_{\nu_{A}}\right)\right], \\
& \Delta_{\tau e}=\frac{4 \Lambda_{\mathrm{L}}^{2}}{v^{4}}\left.-s_{12} c_{12} s_{23} c_{13}\left(m_{\nu_{B}}-m_{\nu_{A}}\right)+c_{23} s_{13} c_{13} e^{i \delta}\left(m_{\nu_{C}}-s_{12}^{2} m_{\nu_{B}}-c_{12}^{2} m_{\nu_{A}}\right)\right], \\
& \Delta_{\tau \mu}=\frac{4 \Lambda_{\mathrm{L}}^{2}}{v^{4}}\left\{s_{23} c_{23}\left[c_{13}^{2} m_{\nu_{C}}+\left(s_{12}^{2} s_{13}^{2}-c_{12}^{2}\right) m_{\nu_{B}}+\left(c_{12}^{2} s_{13}^{2}-s_{12}^{2}\right) m_{\nu_{A}}\right]+\right. \\
&\left.\quad+s_{12} c_{12} s_{13}\left(s_{23}^{2} e^{-i \delta}-c_{23}^{2} e^{i \delta}\right)\left(m_{\nu_{B}}-m_{\nu_{A}}\right)\right\}
\end{aligned}
$$

where, for brevity of notation, $s_{i j}$ and $c_{i j}$ stand for the sine and cosine of the leptonic mixing angles $\theta_{i j}^{\ell}, \delta$ stands for the Dirac CP phase $\delta_{\mathrm{CP}}^{\ell}$, and a generic notation for $\hat{M}_{\nu}$ has been adopted in the definition of $\Delta$ :

$$
\hat{M}_{\nu}^{2} \equiv \operatorname{diag}\left(m_{\nu_{A}}, m_{\nu_{B}}, m_{\nu_{C}}\right)
$$

The three parameters $m_{\nu_{A, B, C}}$ depend on the neutrino mass ordering: for the NO case

$$
m_{\nu_{A}}=0, \quad m_{\nu_{B}}=\Delta m_{\mathrm{sol}}^{2}, \quad m_{\nu_{C}}=\Delta m_{\mathrm{atm}}^{2}
$$

\footnotetext{
${ }^{9}$ The expression for $\Delta$ in the IO case may differ from what reported in ref. [62], due to a different definition taken for the atmospheric mass squared difference.
} 
and for the IO case

$$
m_{\nu_{A}}=\Delta m_{\mathrm{atm}}^{2}-\Delta m_{\mathrm{sol}}^{2}, \quad m_{\nu_{B}}=\Delta m_{\mathrm{atm}}^{2}, \quad m_{\nu_{C}}=0 .
$$

Notice that there is no dependence on the lightest neutrino mass in these expressions. This has an interesting consequence because $\Delta_{i \neq j}$ are completely fixed, apart for the common scale $\Lambda_{\mathrm{L}}$.

2. Extended Field Content I) $\mathcal{G}_{L}=\mathrm{SU}(3)_{\ell_{L}} \times \mathrm{SU}(3)_{e_{R}} \times \mathrm{SO}(3)_{N_{R}} \times C P$. From eqs. (2.24), one gets the following explicit expressions for the off-diagonal entries of $\Delta$ :

$$
\begin{aligned}
\Delta_{\mu e}= & \frac{2 \mu_{\mathrm{L}}}{v^{2}}\left[s_{12} c_{12} c_{23} c_{13}\left(m_{\nu_{B}}-m_{\nu_{A}}\right)+s_{23} s_{13} c_{13} e^{i \delta}\left(e^{-2 i \delta} m_{\nu_{C}}-s_{12}^{2} m_{\nu_{B}}-c_{12}^{2} m_{\nu_{A}}\right)\right], \\
\Delta_{\tau e}= & \frac{2 \mu_{\mathrm{L}}}{v^{2}}\left[-s_{12} c_{12} s_{23} c_{13}\left(m_{\nu_{B}}-m_{\nu_{A}}\right)+c_{23} s_{13} c_{13} e^{i \delta}\left(e^{-2 i \delta} m_{\nu_{C}}-s_{12}^{2} m_{\nu_{B}}-c_{12}^{2} m_{\nu_{A}}\right)\right], \\
\Delta_{\tau \mu}= & \frac{2 \mu_{\mathrm{L}}}{v^{2}}\left\{s_{23} c_{23}\left(c_{13}^{2} m_{\nu_{C}}-c_{12}^{2} m_{\nu_{B}}-s_{12}^{2} m_{\nu_{A}}\right)+\right. \\
& \left.+s_{12} c_{12} s_{13} e^{i \delta}\left(s_{23}^{2}-c_{23}^{2}\right)\left(m_{\nu_{B}}-m_{\nu_{A}}\right)+s_{23} c_{23} s_{13}^{2} e^{2 i \delta}\left(s_{12}^{2} m_{\nu_{B}}+c_{12}^{2} m_{\nu_{A}}\right)\right\}, \quad \text { (3.9) }
\end{aligned}
$$

where a generic notation — different from the one in the MFC case - for $\hat{M}_{\nu}$ has been adopted:

$$
\hat{M}_{\nu} \equiv \operatorname{diag}\left(m_{\nu_{A}}, m_{\nu_{B}}, m_{\nu_{C}}\right) .
$$

The three parameters $m_{\nu_{A, B, C}}$ are now defined by

$$
m_{\nu_{A}}=m_{\nu_{1}}, \quad m_{\nu_{B}}=e^{i \alpha_{21}} \sqrt{\Delta m_{\mathrm{sol}}^{2}+m_{\nu_{1}}^{2}}, \quad m_{\nu_{C}}=e^{i \alpha_{31}} \sqrt{\Delta m_{\mathrm{atm}}^{2}+m_{\nu_{1}}^{2}},
$$

for the NO case, $m_{\nu_{1}}<m_{\nu_{2}}<m_{\nu_{3}}$, and by

$$
m_{\nu_{A}}=\sqrt{\Delta m_{\mathrm{atm}}^{2}-\Delta m_{\mathrm{sol}}^{2}+m_{\nu_{3}}^{2}}, \quad m_{\nu_{B}}=e^{i \alpha_{21}} \sqrt{\Delta m_{\mathrm{atm}}^{2}+m_{\nu_{3}}^{2}}, \quad m_{\nu_{C}}=e^{i \alpha_{31}} m_{\nu_{3}},
$$

for the IO case, $m_{\nu_{3}}<m_{\nu_{1}}<m_{\nu_{2}}$.

The hypothesis of CP conservations fixes the Dirac and Majorana CP phases to be $\delta=\{0, \pi\}$ and $\alpha_{21,31}=0$ in these expressions. Indeed, while $\Delta_{i j}$ would be real even for $\alpha_{21,31}=\pi$ and therefore no CPV process would be described with $\Delta$ insertions, $Y_{\nu}$ would be complex and then it would not be possible to express the spurions insertions in eq. (2.23) in terms of low-energy parameters, losing the predictivity power of MLFV.

In the strong hierarchical limit, $m_{\nu_{1}} \ll m_{\nu_{2}}<m_{\nu_{3}}$ in the NO case and $m_{\nu_{3}} \ll m_{\nu_{1}}<m_{\nu_{2}}$ in the IO one, and setting the lightest neutrino mass to zero, the expressions for $m_{\nu_{A, B, C}}$ reduce to the square root of those for the MFC case, as can be deduced comparing eqs. (3.6) and (3.10), and the results for $\Delta_{i \neq j}$ get simplified. Also in this case, only one parameter remains free, that is the LNV scale $\mu_{\mathrm{L}}$.

When the neutrino mass hierarchy is milder or the eigenvalues are almost degenerate, the lightest neutrino mass cannot be neglected and represents a second free parameters of $\Delta_{i \neq j}$, besides $\mu_{\mathrm{L}}$. 
3. Extended Field Content II) $\mathcal{G}_{L}=\mathrm{SU}(3)_{\ell_{L}+N_{R}} \times \mathrm{SU}(3)_{e_{R}}$. The expressions for the offdiagonal entries of $\Delta$ that follow from eqs. (2.29) can be obtained from the expressions in eq. (3.5), by substituting

$$
\frac{4 \Lambda_{\mathrm{L}}^{2}}{v^{4}} \rightarrow \frac{v^{4}}{4 \mu_{\mathrm{L}}^{2}}
$$

and taking the following notation for $\hat{M}_{\nu}$ :

$$
\hat{M}_{\nu}^{-2} \equiv \operatorname{diag}\left(m_{\nu_{A}}, m_{\nu_{B}}, m_{\nu_{C}}\right)
$$

with $m_{\nu_{A, B, C}}$ given by

$$
m_{\nu_{A}}=\frac{1}{m_{\nu_{1}}^{2}}, \quad m_{\nu_{B}}=\frac{1}{\Delta m_{\mathrm{sol}}^{2}+m_{\nu_{1}}^{2}}, \quad m_{\nu_{C}}=\frac{1}{\Delta m_{\mathrm{atm}}^{2}+m_{\nu_{1}}^{2}},
$$

for the NO case, and

$$
m_{\nu_{A}}=\frac{1}{\Delta m_{\mathrm{atm}}^{2}-\Delta m_{\mathrm{sol}}^{2}+m_{\nu_{3}}^{2}}, \quad m_{\nu_{B}}=\frac{1}{\Delta m_{\mathrm{atm}}^{2}+m_{\nu_{3}}^{2}}, \quad m_{\nu_{C}}=\frac{1}{m_{\nu_{3}}},
$$

for the IO case.

The limits for the lightest neutrino mass being zero are not well defined for this case, as it would lead to an infinity in the expressions for $\Delta_{i \neq j}$. Differently from the other two cases, only a moderate neutrino mass hierarchy is then allowed. Finally, these expressions depend on two free parameters, the lightest neutrino mass and the LNV scale $\mu_{\mathrm{L}}$

\subsection{Rare radiative leptonic decays and conversion in nuclei}

In the formalism of the effective Lagrangian reported in the eq. (3.2), the Beyond SM (BSM) contributions to the branching ratio of leptonic radiative rare decays are given by

$$
B_{\ell_{i} \rightarrow \ell_{j} \gamma} \equiv \frac{\Gamma\left(\ell_{i} \rightarrow \ell_{j} \gamma\right)}{\Gamma\left(\ell_{i} \rightarrow \ell_{j} \nu_{i} \bar{\nu}_{j}\right)}=384 \pi^{2} e^{2} \frac{v^{4}}{4 \Lambda_{\mathrm{LFV}}^{4}}\left|\Delta_{i j}\right|^{2}\left|c_{R L}^{(2)}-c_{R L}^{(1)}\right|^{2},
$$

being $e$ the electric charge, and where the corrections of the Wilson coefficient due to the electroweak renormalisation from the scale of NP down to the mass scale of the interested lepton $[199,200]$ have been neglected, and the limit $m_{\ell_{j}} \ll m_{\ell_{i}}$ has been taken.

The same contributions to the branching ratio for $\mu \rightarrow e$ conversion in a generic nucleus of mass number $A$ read

$$
\begin{aligned}
B_{\mu \rightarrow e}^{A}= & \frac{32 G_{F}^{2} m_{\mu}^{5}}{\Gamma_{\mathrm{capt}}^{A}} \frac{v^{4}}{4 \Lambda_{\mathrm{LFV}}^{4}}\left|\Delta_{\mu e}\right|^{2} \mid\left(\left(\frac{1}{4}-s_{w}^{2}\right) V^{(p)}-\frac{1}{4} V^{(n)}\right)\left(c_{L L}^{(1)}+c_{L L}^{(2)}\right)+ \\
& +\frac{3}{2}\left(V^{(p)}+V^{(n)}\right) c_{L L}^{(3)}+\left(V^{(p)}+\frac{1}{2} V^{(n)}\right) c_{L L}^{(4 u)}+\left(\frac{1}{2} V^{(p)}+V^{(n)}\right) c_{L L}^{(4 d)}+ \\
& +\frac{1}{2}\left(-V^{(p)}+V^{(n)}\right) c_{L L}^{(5)}-\left.\frac{e D_{A}}{4}\left(c_{R L}^{(2)}-c_{R L}^{(1)}\right)^{*}\right|^{2}
\end{aligned}
$$




\begin{tabular}{|c|c|c|c|c|}
\hline & $V^{(p)}$ & $V^{(p)}$ & $D$ & $\Gamma_{\text {capt }}\left(10^{6} \mathrm{~s}^{-1}\right)$ \\
\hline $\mathrm{Au}$ & 0.0974 & 0.146 & 0.189 & 13.07 \\
$\mathrm{Al}$ & 0.0161 & 0.0173 & 0.0362 & 0.7054 \\
\hline
\end{tabular}

Table 2. Reference values for nuclear overlap integrals and capture rates from ref. [201].

where $s_{W} \equiv \sin \theta_{W}=0.23, V^{(p)}, V^{(n)}$ and $D$ are dimensionless nucleus-dependent overlap integrals that can be found in table 2 for Aluminium and Gold, that also contains the numerical values for decay rate of the muon capture, which has been used to normalise the decay rate for the $\mu \rightarrow e$ conversion.

The experimental bounds on these processes that will be considered in the numerical analysis are the following:

$$
\begin{aligned}
B_{\mu \rightarrow e \gamma} & <5.7 \times 10^{-13}[202]\left(6 \times 10^{-14}[203]\right), \\
B_{\tau \rightarrow e \gamma} & <5.2 \times 10^{-8}[204]\left(10^{-9} \div 10^{-10}[205]\right), \\
B_{\tau \rightarrow \mu \gamma} & <2.5 \times 10^{-7}[204]\left(10^{-8} \div 10^{-9}[205]\right), \\
B_{\mu \rightarrow e}^{\mathrm{Au}} & <7 \times 10^{-13}[206], \\
B_{\mu \rightarrow e}^{\mathrm{Al}} & <6 \times 10^{-17}[207,208],
\end{aligned}
$$

where the values in the brackets and the bound on $B_{\mu \rightarrow e}^{\mathrm{Al}}$ refer to future expected sensitivities.

\subsubsection{Bounds on the LFV scale}

The bounds on the LNV scales, determined in eqs. (2.15), (2.25) and (2.30), can be translated into bounds on the LFV scale when considering the experimental limits in the rare processes introduced above. Indeed, after substituting the expressions for $\Delta$, defined in eqs. (2.16), (2.24) and (2.29), into the eqs. (3.17) and (3.18), one can rewrite these expressions extracting the dependence on the NP scales:

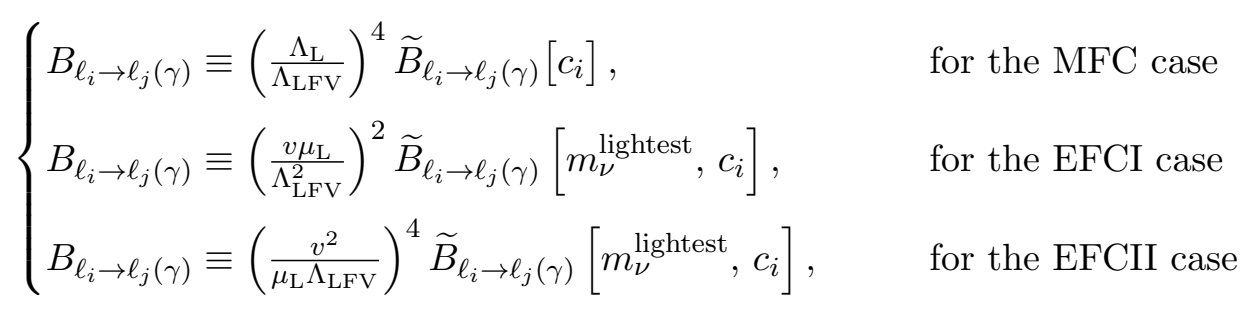

where the square brackets list the free parameters, that is the lightest neutrino mass (only for the EFCI and EFCII cases) and the effective Lagrangian parameters $c_{i}$.

The numerical analysis reveals that the strongest bounds on the $\Lambda_{\mathrm{LFV}}$ comes from the data on $\mu \rightarrow e$ conversion in gold, although similar results are provided by the data on leptonic radiative rare decays. The corresponding parameter space is shown in figure 1 , obtained taking the best fit values for the quantities in table 1 (for the EFCI case, the Dirac CP phase can only acquire two values, 0 and $\pi$ ) and the data from table 2 . Although these plots have been generated for the NO neutrino spectrum, they hold for the IO case 

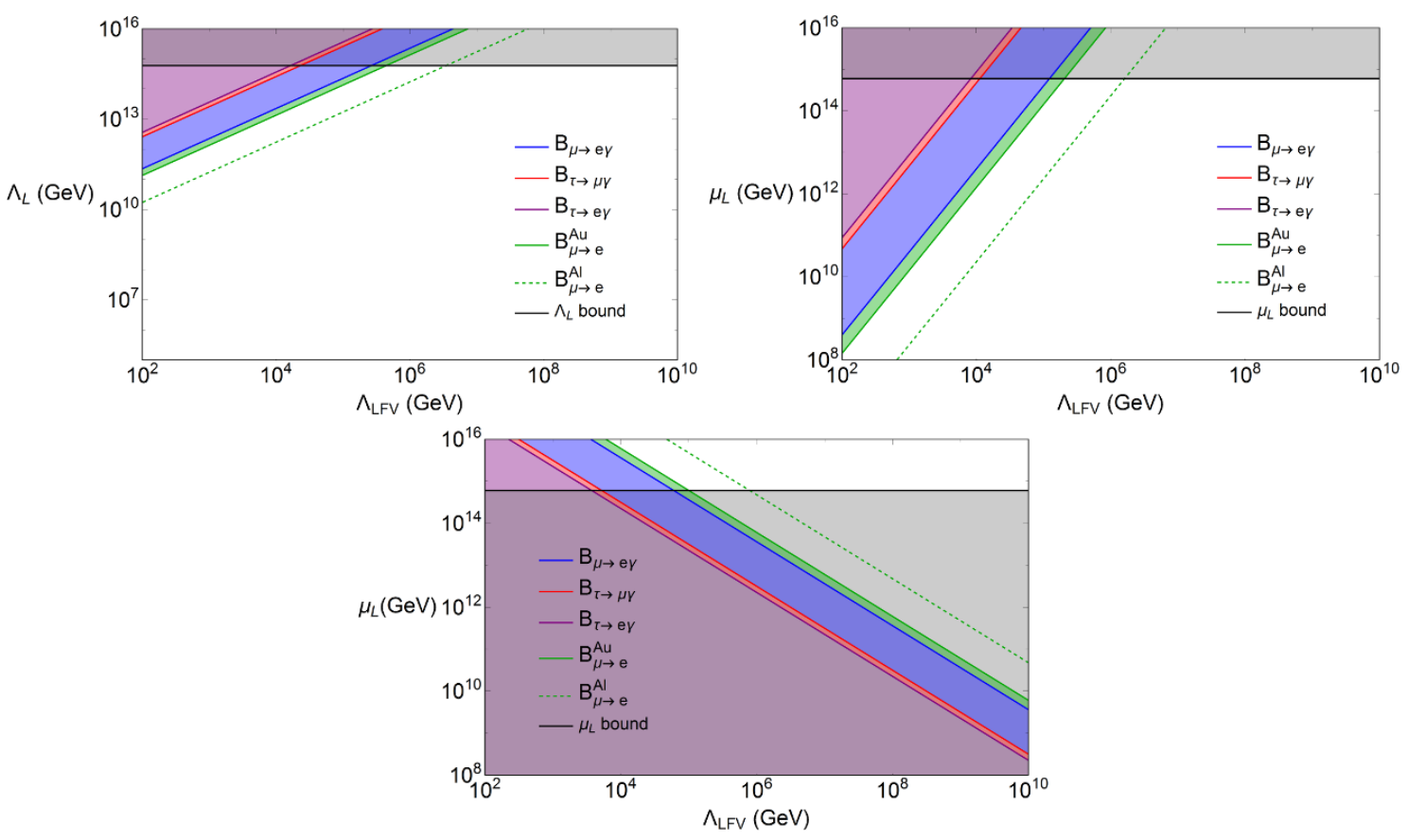

Figure 1. Parameter space for the LFV and LNV scales constrained by requiring perturbativity of the spurion backgrounds and by the present experimental bounds on $\mu \rightarrow e$ conversion in gold (in green), $B R(\mu \rightarrow e \gamma)$ (in blue), $B R(\tau \rightarrow \mu \gamma)$ (in red), and $B R(\tau \rightarrow e \gamma)$ (in purple). Taking into account the expected future sensitivity on $B R(\mu \rightarrow e \gamma)$ would not restrict further the parameter space in the case of a negative result: the prospective bound would almost coincide with the bound from the negative search for $\mu \rightarrow e$ conversion in gold, $B R(\mu \rightarrow e)$. However, with the planned significant increase (by more than 4 orders of magnitude) of the sensitivity to the relative rate of $\mu \rightarrow e$ conversion in aluminium it would be possible to probe considerably larger fraction of the parameter space of interest: the corresponding bound is drown as the green dashed line. The grey region are excluded areas from the constraints on the LNV scale, eqs. (2.15), (2.25), and (2.30). The left, middle and right panels correspond to the MFC, EFCI and EFCII cases, respectively. The border lines are obtained taking as input data the best fit values for the oscillation parameters listed in table 1 and the nuclear quantities in table 2. The Dirac CP phase for the EFCI plot is set equal to $\pi$, while the Majorana are set to 0 , in order to minimise the excluded region of the parameter space. For the EFCI and EFCII cases, a quasi-degenerate neutrino mass spectrum with $m_{\nu}^{\text {lightest }}=0.1 \mathrm{eV}$ has been assumed, which also minimised the excluded areas. In all the cases, the Lagrangian coefficients have been fixed in a democratic way not to favour any specific operator contribution: $c_{L L}^{(1)}+c_{L L}^{(2)}=1=c_{L L}^{(3)}=c_{L L}^{(4 u)}=c_{L L}^{(4 d)}=c_{L L}^{(5)}=c_{R L}^{(2)}-c_{R L}^{(1)}$.

as well, as no difference is appreciable. On the other hand, a dependence on the strength of the splitting between neutrino masses can be found for the EFC scenarios: the plots reported here illustrate the almost degenerate case, where the lightest neutrino mass is taken to be $\mathcal{O}(0.1 \mathrm{eV})$; stronger hierarchies result in a more constrained parameter space. Finally, the plot for EFCI refers to $\delta_{\mathrm{CP}}^{\ell}=\pi$, but the other case with $\delta_{\mathrm{CP}}^{\ell}=0$ is almost indistinguishable. 
The upper bound on $\Lambda_{\mathrm{L}}$ for the MFC case reduce the parameter space, although it cannot be translated into upper bounds on $\Lambda_{\mathrm{LFV}}$ : larger $\Lambda_{\mathrm{LFV}}$ simply further suppresses the expected values for the branching ratios of the observables considered. Moreover, no lower bound can be drown: requiring to close the experimental bound for the $\mu \rightarrow e$ conversion, small $\Lambda_{\mathrm{LFV}}$ requires small $\Lambda_{\mathrm{L}}$, leading at the same time to tune $g_{\nu}$ to small values, in order to reproduce the correct masses for the light active neutrinos, see eq. (2.12). The same occurs for EFCI, for $\mu_{\mathrm{L}}$ and $Y_{\nu}$, although, in this case, this can be well justified considering the additional Abelian symmetries appearing in eq. (2.19), as discussed in ref. [65]. When considering the EFCII case, the lower bound on $\Lambda_{\mathrm{L}}$ removes a large part of the parameter space, but does not translate into a lower bound on $\Lambda_{\mathrm{LFV}}$ : for example, for $\Lambda_{\mathrm{L}}$ at its lower bound in eq. (2.30), $\Lambda_{\mathrm{LFV}}$ must be larger than $10^{5} \mathrm{GeV}$ in order to satisfy to the present bounds on $B_{\mu \rightarrow e}^{\mathrm{Au}}$; however, for larger values of $\Lambda_{\mathrm{L}}, \Lambda_{\mathrm{LFV}}$ can be smaller, down to the TeV scale for $\Lambda_{\mathrm{L}} \sim 10^{17} \mathrm{GeV}$, although in this case a tuning on $\left|Y_{N}\right|$ is necessary in order to reproduce correctly the lightness of the active neutrino masses.

The absence of evidence of NP in direct and indirect searches at colliders and lowenergy experiments suggests that NP leading to LFV should be heavier than a few $\mathrm{TeV}$. In the optimistic scenario that NP is just behind the corner and waiting to be discovered in the near future, an indication of the LNV scale could be extracted from the plots in figure 1. Indeed, if $\mu \rightarrow e$ conversion in nuclei is observed, $\Lambda_{\mathrm{LFV}} \sim 10^{3} \div 10^{4} \mathrm{GeV}$ will lead to $\Lambda_{\mathrm{L}} \sim 10^{12} \div 10^{13} \mathrm{GeV}$ for MFC, $\mu_{\mathrm{L}} \sim 10^{9} \div 10^{10} \mathrm{GeV}$ for EFCI, and $\mu_{\mathrm{L}} \sim 10^{16} \div 10^{17} \mathrm{GeV}$ for EFCII. In the EFC scenarios, the LNV scale is associated to the masses of the RH neutrinos, that therefore turn out to be much heavier than the energies reachable at present and future colliders. An exception is the case where additional Abelian factors are considered in the flavour symmetry that allows to separate the LNV scale and the RH neutrino masses [65]: this opens the possibility of producing sterile neutrinos at colliders and then of studying their interactions in direct searches.

\subsubsection{Ratios of branching ratios}

The information encoded in eq. (3.20) are not limited to the scales of LFV and LNV. Studying the ratios of branching ratios between the different processes reveals characteristic features that may help to disentangle the different versions of MLFV. To shorten the notation,

$$
R_{i \rightarrow j \gamma}^{t \rightarrow s \gamma} \equiv \frac{\widetilde{B}_{\ell_{t} \rightarrow \ell_{s} \gamma}}{\widetilde{B}_{\ell_{i} \rightarrow \ell_{j} \gamma}}
$$

will be adopted in the analysis that follows. These observables do not depend on the LFV and LNV scales, nor on the Lagrangian coefficients. They are sensible to the neutrino oscillation parameters and, for the EFC cases, to the mass of the lightest active neutrino. For MFC, they do not even depend on $m_{\nu}^{\text {lightest }}$ : although the corresponding plots only contain points along an horizontal line, they will be reported in the next subsections in order to facilitate the comparison with the other cases.

The two branching ratios with the best present sensitivities, the one for $\mu \rightarrow e$ conversion in nuclei and the one for $\mu \rightarrow e \gamma$, have the same dependence on $\Delta_{\mu e}$ and therefore 


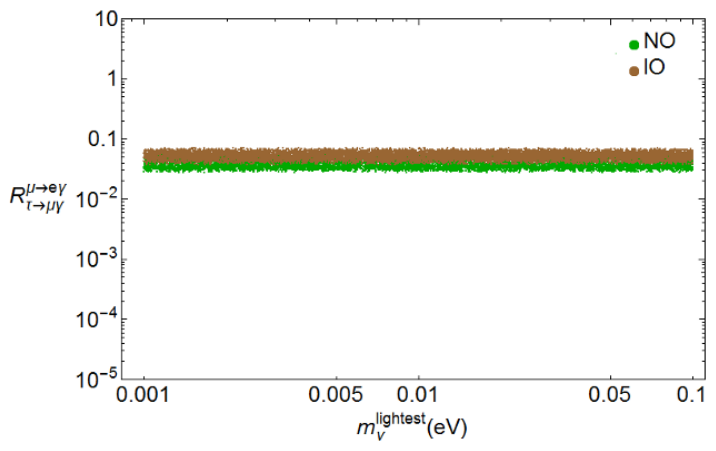

(a) MFC

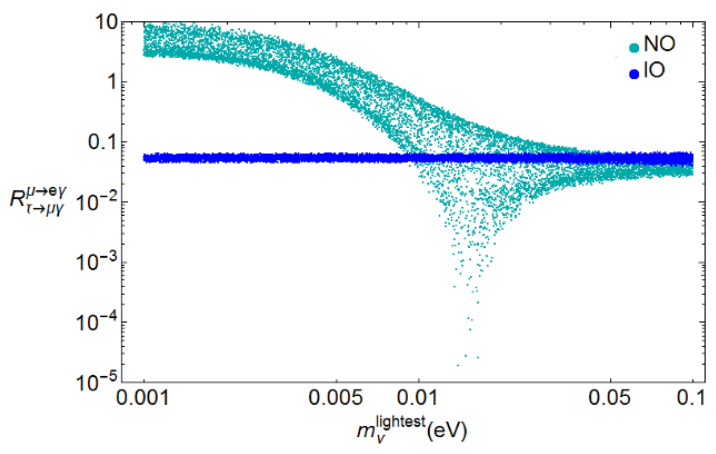

(c) EFCII

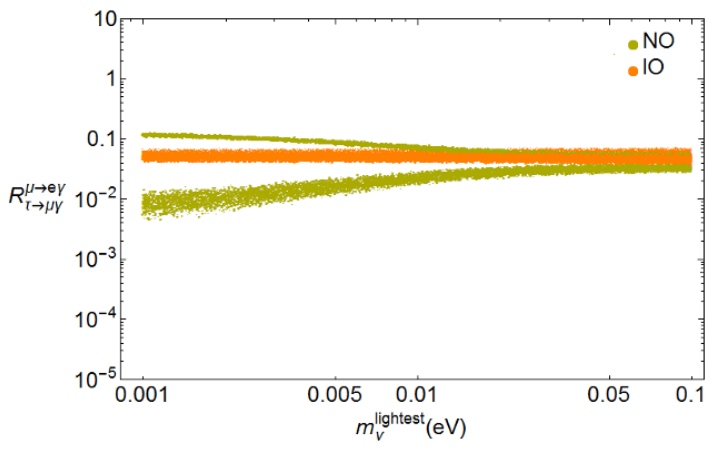

(b) EFCI

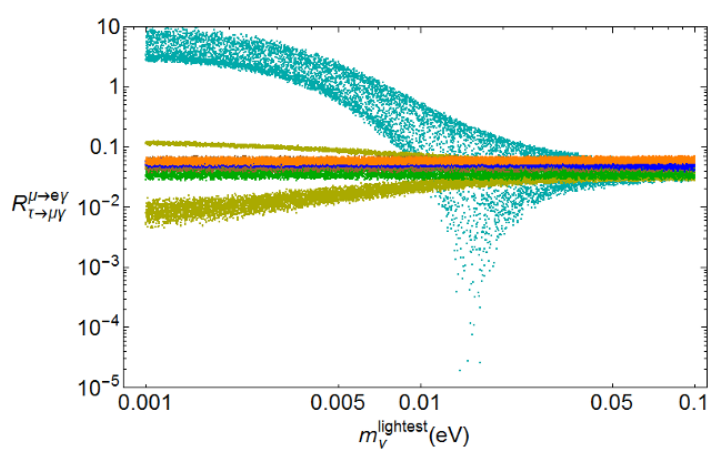

(d) All Cases

Figure 2. $R_{\tau \rightarrow \mu \gamma}^{\mu \rightarrow e \gamma}$ for the MFC, EFCI and EFCII from upper left to lower left. Lower right reports the previous plots altogether. Colour codes can be read directly on each plot.

their ratio is not sensitive to the charged lepton and neutrino masses and to the neutrino mixing. Instead, as pointed out in ref. [76], this ratio may be sensitive to the chirality of the effective operators contributing to these observables. The comparison between eqs. (3.17) and (3.18) shows that only $B_{\mu \rightarrow e}^{A}$ is sensitive to $\mathcal{O}_{L L}^{(i)}$, and thus any deviation from

$$
\frac{B_{\mu \rightarrow e}^{A}}{B_{\mu \rightarrow e \gamma}}=\pi D_{A}^{2}
$$

would be a signal of this set of operators.

In the scatter plots that follow, neutrino oscillation parameters are taken from table 1 as random values inside their $2 \sigma$ error bands. The lightest neutrino mass is taken in the range $m_{\nu}^{\text {lightest }} \subset[0.001,0.1] \mathrm{eV}$ and the results for the NO and IO spectra are shown with different colours. In these figures, the density of the points should not be interpreted as related to the likelihood of differently populated regions of the parameter space.

$\boldsymbol{R}_{\tau \rightarrow \boldsymbol{\mu} \gamma}^{\boldsymbol{\mu} \rightarrow \boldsymbol{e \gamma}}$. In the upper left, upper right and lower left panes in figure 2, the results are reported for the ratio of the branching ratios of the $\mu \rightarrow e \gamma$ and $\tau \rightarrow \mu \gamma$ decays for the MFC, EFCI and EFCII cases, respectively. Figure 2d is a summarising figure where all the three plots are shown together to facilitate the comparison and to make clearer the non-overlapping areas. 
As figure 2a shows, $R_{\tau \rightarrow \mu \gamma}^{\mu \rightarrow e \gamma}$ is independent of the lightest neutrino mass. The two sets of points corresponding to NO and IO spectra almost overlap, making it very hard to distinguish between the two neutrino mass orderings.

In figure $2 \mathrm{~b}$, the dependence on $m_{\nu}^{\text {lightest }}$ can be slightly appreciated and the predictions for two mass orderings do not overlap when the spectrum is hierarchical. In the NO case there are two branches associated with the two values of $\delta_{\mathrm{CP}}^{\ell}$ : the values associated with the $\delta_{\mathrm{CP}}^{\ell}=0$-branch are very close to those for the IO spectrum and correspond to the positive sum of the two terms on the right-hand side of eq. (3.9); the values associated with the $\delta_{\mathrm{CP}}^{\ell}=\pi$-branch are smaller by about one order of magnitude, which reflects a partial cancellation between the two terms in the right-hand side of eq. (3.9). In the IO case there is only one branch because the first term on the right-hand side of eq. (3.9) is dominant.

As figure $2 \mathrm{c}$ shows, the points for the two mass orderings overlap in the quasidegenerate limit down to masses of about $0.05 \mathrm{eV}$. However, they show different profiles in the hierarchical limit. In the IO case the ratio of branching ratios under discussion is almost constant with $m_{\nu}^{\text {lightest }}$. In the NO case the ratio $R_{\tau \rightarrow \mu \gamma}^{\mu \rightarrow e \gamma}$ can be as small as few $\times 10^{-4}$ at $\sim 0.012 \mathrm{eV}$, while for $m_{\nu 1}<0.01 \mathrm{eV}$ the ratio is $R_{\tau \rightarrow \mu \gamma}^{\mu \rightarrow e \gamma}>1$. As discussed in ref. [65], this can be understood from eqs. (3.5) and (3.15): in the NO case and strong mass hierarchy, the dominant contribution is proportional to $1 / m_{\nu_{1}}$ and therefore $R_{\tau \rightarrow \mu \gamma}^{\mu \rightarrow e \gamma}$ gets enhanced; while when the spectrum is almost degenerate and in the IO case, the dominant contribution is suppressed by the sine of the reactor angle and the dependence on the lightest neutrino mass is negligible.

In figure $2 \mathrm{~d}$, where the three cases are shown altogether, it can be seen that all the cases overlap for the IO spectrum and in the quasi-degenerate limit for the NO spectrum, predicting $R_{\tau \rightarrow \mu \gamma}^{\mu \rightarrow e \gamma} \cong 0.02 \div 0.07$. When the mass spectrum is of NO type and hierarchical, the ratio spans values from 0.004 to 10 . Interestingly, if this ratio is observed to be larger than 0.1, or smaller than 0.004, then only the EFCII with NO spectrum can explain it. Notice that, given the current limits on $B_{\mu \rightarrow e \gamma}$, values smaller than $\sim 6 \times 10^{-4}$ would be testable in the future planned experiments searching for $\tau \rightarrow \mu \gamma$.

$\boldsymbol{R}_{\tau \rightarrow e \gamma}^{\mu \rightarrow e \gamma}$. The ratio $R_{\tau \rightarrow e \gamma}^{\mu \rightarrow e \gamma}$ exhibits features which are very similar to those of the ratio $R_{\tau \rightarrow \mu \gamma}^{\mu \rightarrow e \gamma}$. Figures $3 \mathrm{a}$ and $3 \mathrm{~b}$ are very similar to figures $2 \mathrm{a}$ and $2 \mathrm{~b}$ : the profiles of the points are the same, only the area spanned is different, as indeed $R_{\tau \rightarrow e \gamma}^{\mu \rightarrow e \gamma}$ is predicted to be by almost one order of magnitude larger than $R_{\tau \rightarrow \mu \gamma}^{\mu \rightarrow e \gamma}$. Similar conclusions, however, apply. Figure 3c, instead, shows an interesting difference with respect to its sibling figure $2 \mathrm{c}$ : the IO and the NO points cover almost the same nearly horizontal area both for quasi-degenerate masses and for a hierarchical mass spectrum, the NO region being slightly wider. Only for values of the lightest neutrino mass between $0.01 \mathrm{eV}$ and $0.02 \mathrm{eV}$, there could be an enhancement or a suppression of $R_{\tau \rightarrow e \gamma}^{\mu \rightarrow e \gamma}$ in the EFCII case. This is a distinctive feature that could allow to disentangle EFCII from the other cases: values of $R_{\tau \rightarrow e \gamma}^{\mu \rightarrow e \gamma}$ larger than 10 or smaller than 0.04 can only be explained by a NO neutrino spectrum in the case of EFCII. Notice that, given the current limits on $B_{\mu \rightarrow e \gamma}$, values smaller than 0.006 would be testable in the future planned experiments searching for $\tau \rightarrow e \gamma$. 


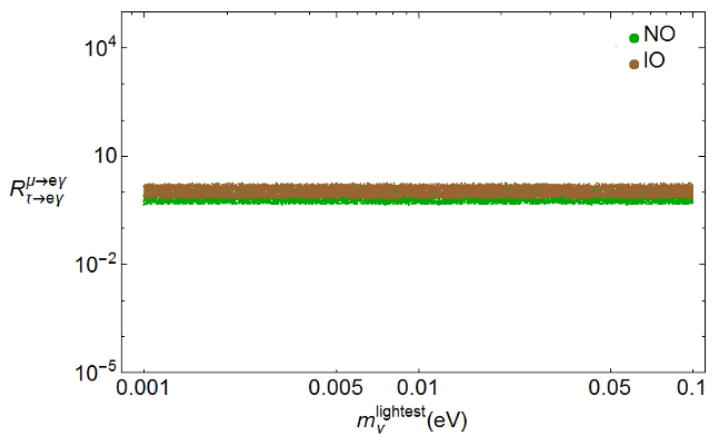

(a) MFC

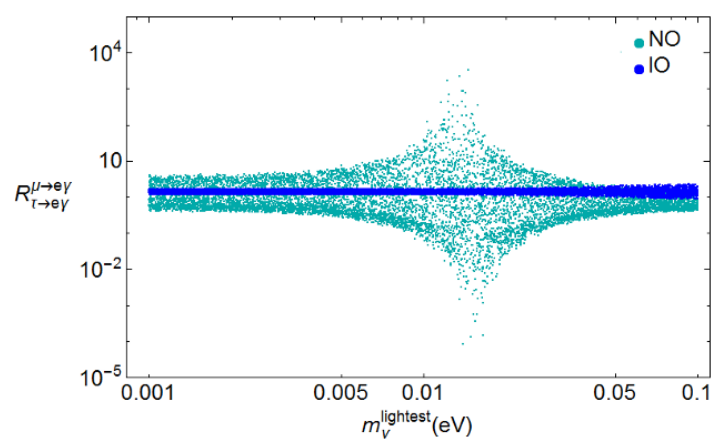

(c) EFCII

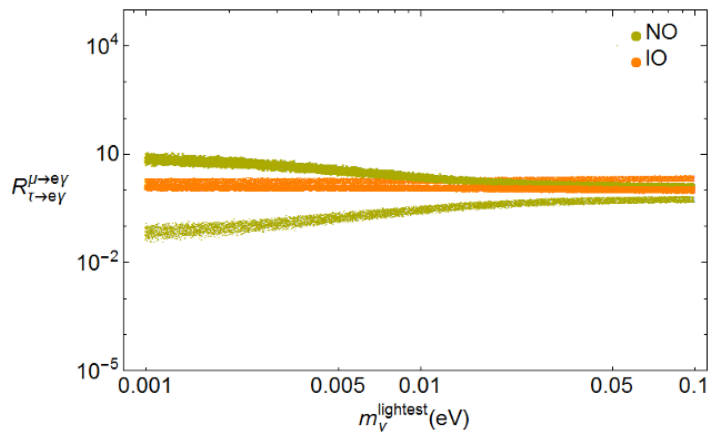

(b) EFCI

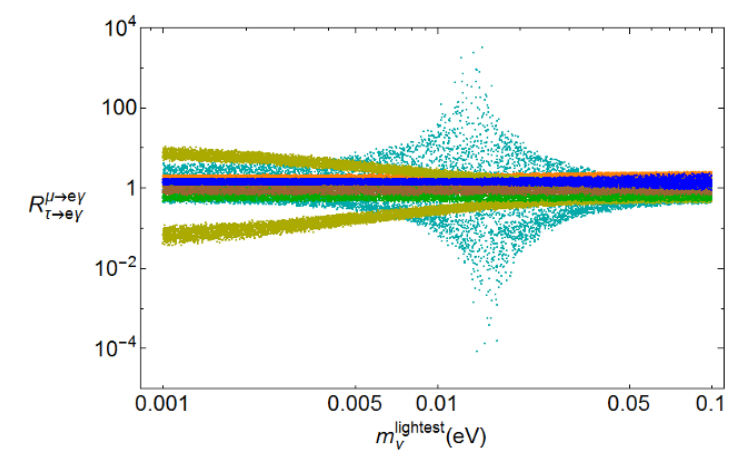

(d) All Cases

Figure 3. $R_{\tau \rightarrow e \gamma}^{\mu \rightarrow e \gamma}$ for the MFC, EFCI and EFCII from upper left to lower left. Lower right reports the previous plots altogether. Colour codes can be read directly on each plot.

$\boldsymbol{R}_{\tau \rightarrow \boldsymbol{\mu} \gamma}^{\boldsymbol{\tau} \rightarrow \boldsymbol{e}}$. The ratio $R_{\tau \rightarrow \mu \gamma}^{\tau \rightarrow e \gamma}$ is almost indistinguishable form the ratio $R_{\tau \rightarrow \mu \gamma}^{\mu \rightarrow e \gamma}$ except for the EFCII case with NO neutrino mass spectrum. For the other cases the conclusions for $R_{\tau \rightarrow \mu \gamma}^{\tau \rightarrow e \gamma}$ are almost the same as the conclusions reached for $R_{\tau \rightarrow \mu \gamma}^{\mu \rightarrow e \gamma}$. One can see that values for $R_{\tau \rightarrow \mu \gamma}^{\tau \rightarrow e \gamma}$ smaller than 0.01 or larger than 0.1 would only be explain by EFCII with NO neutrino spectrum.

Summarising, the study of these three ratios can provide relevant information if values for these ratios are found to be larger than 0.1 (10) for $R_{\tau \rightarrow \mu \gamma}^{\mu \rightarrow e \gamma}$ and $R_{\tau \rightarrow \mu \gamma}^{\tau \rightarrow e \gamma}$ (for $R_{\tau \rightarrow e \gamma}^{\mu \rightarrow e \gamma}$ ) or smaller than 0.004 for $R_{\tau \rightarrow \mu \gamma}^{\mu \rightarrow e \gamma}, 0.01$ for $R_{\tau \rightarrow \mu \gamma}^{\tau \rightarrow e \gamma}$, and 0.04 for $R_{\tau \rightarrow e \gamma}^{\mu \rightarrow e \gamma}$ : such values can be explained only in the case of EFCII with NO spectrum. If large values for $R_{\tau \rightarrow \mu \gamma}^{\mu \rightarrow e \gamma}$ and $R_{\tau \rightarrow \mu \gamma}^{\tau \rightarrow e \gamma}$ are found, then this would point to a relatively small value for the lightest neutrino mass, smaller than $0.008 \mathrm{eV}$; this should occur consistently with a value for $R_{\tau \rightarrow e \gamma}^{\mu \rightarrow e \gamma}$ between 0.1 and 10. If instead, $R_{\tau \rightarrow e \gamma}^{\mu \rightarrow e \gamma}$ is found to be much larger than 10, this would imply masses for the lightest neutrino between $0.008 \mathrm{eV}$ and $0.04 \mathrm{eV}$; consistently, $R_{\tau \rightarrow \mu \gamma}^{\mu \rightarrow e \gamma}$ and $R_{\tau \rightarrow \mu \gamma}^{\tau \rightarrow e \gamma}$ should remain smaller than 1 . Finally, if no signals are seen in all the three ratios and bounds of 0.004 (0.01) [0.04] or smaller can be obtained for $R_{\tau \rightarrow \mu \gamma}^{\mu \rightarrow e \gamma}\left(R_{\tau \rightarrow \mu \gamma}^{\tau \rightarrow e \gamma}\right)\left[R_{\tau \rightarrow e \gamma}^{\mu \rightarrow e \gamma}\right.$, then this would be consistent with masses between $0.01 \mathrm{eV}$ and $0.02 \mathrm{eV}$ for the lightest neutrino, or otherwise MLFV cannot explain this feature. On the other hand, all the three MLFV versions, for both the mass orderings, can explain values for these ratios inside the regions 


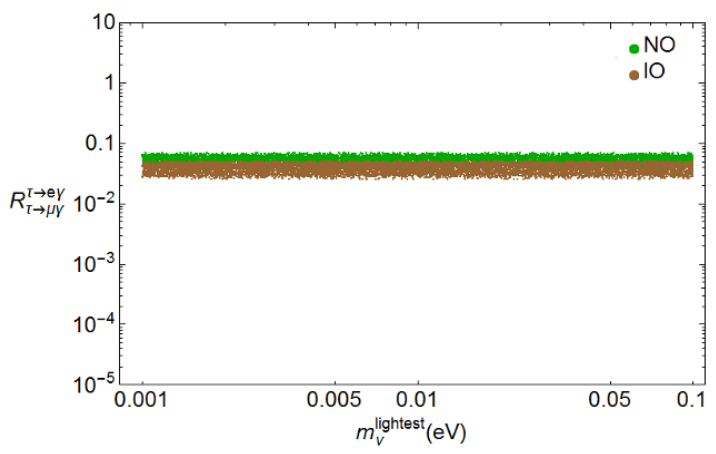

(a) MFC

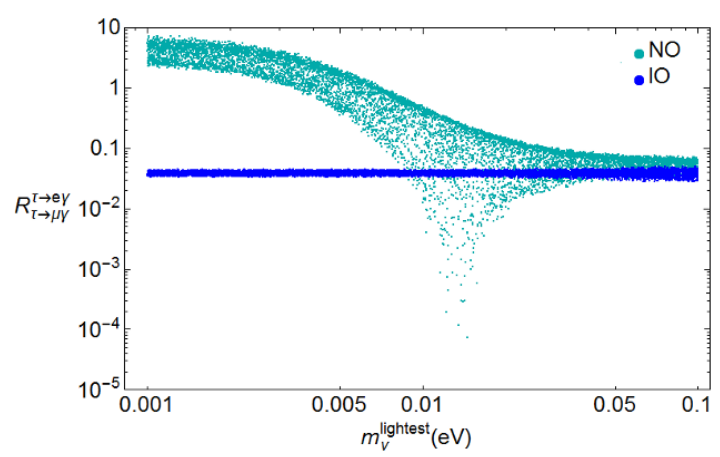

(c) EFCII

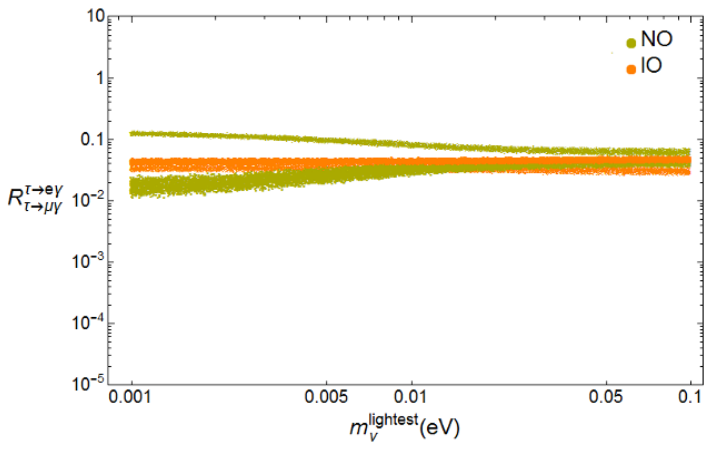

(b) EFCI

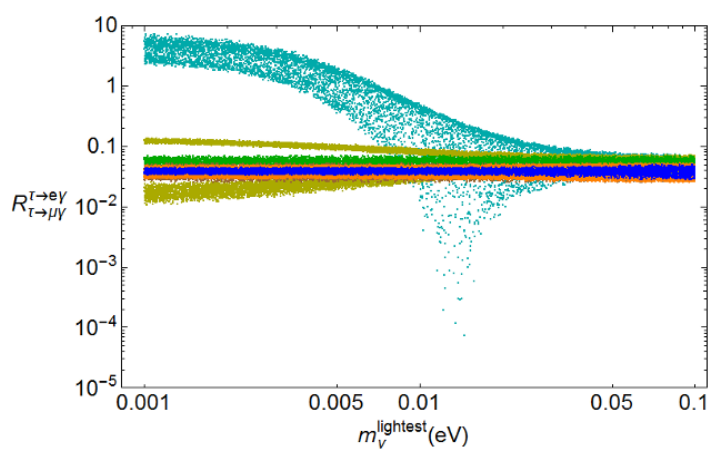

(d) All Cases

Figure 4. $R_{\tau \rightarrow \mu \gamma}^{\tau \rightarrow e \gamma}$ for the MFC, EFCI and EFCII from upper left to lower left. Lower right reports the previous plots altogether. Colour codes can be read directly on each plot.

aforementioned, generally between 0.01 and 0.1 : this case would be the less favourable for distinguishing the different setups.

These results are generically in agreement with previous analyses performed in refs. $[62,63,65,76]$ and the differences are due to the update input data used here.

$\boldsymbol{B}_{\boldsymbol{\mu} \rightarrow e^{\cdot}}^{\mathbf{A}} \quad$ As shown in eq. (3.22), the ratio of the two branching ratios with the best present sensitivities is independent from $\Delta$ and can be used to obtain information about the chirality of the operators contributing to the $\mu \rightarrow e$ conversion process. On the other hand, if the observation (or non-observation) of the leptonic radiative rare decays allows to identify the MLFV realisation from figures 2, 3 and 4, the branching ratio of the $\mu \rightarrow e$ conversion in nuclei could provide the missing information necessary to fix the LFV scale. As an example, one can assume that an upper bound on $R_{\tau \rightarrow \mu \gamma}^{\mu \rightarrow e \gamma}$ of about 0.004 has been set, that could be explained by EFCII with a NO neutrino spectrum and a mass of the lightest neutrino of about $0.014 \mathrm{eV}$. The upper bound on $B_{\mu \rightarrow e}^{\mathrm{Au}}$ implies the upper bound $v^{2} /\left(\mu_{\mathrm{L}} \Lambda_{\mathrm{LFV}}\right)<5.7 \times 10^{-17}$. By fixing the LNV scale to its lower bound, one finds that these observables can provide information on the LFV scale that should be larger than about $2 \times 10^{6} \mathrm{GeV}$. The future expected sensitivity on $B_{\mu \rightarrow e}^{\mathrm{Al}}$ is better than the presently achieved one by four orders of magnitude. A negative results of the planned future searches for $\mu \rightarrow e$ conversion would imply a bound on the LFV scale of about $10^{7} \mathrm{GeV}$. 


\section{$4 \quad b \rightarrow s$ anomalies}

The effective Lagrangian in eq. (3.2) contains the operators which provide the most relevant contributions to the $b \rightarrow s$ anomalies under discussion: ${ }^{10}$ they are $\mathcal{O}_{L L}^{(3)}$ and $\mathcal{O}_{L L}^{(5)}$, which contribute at tree level to the Wilson coefficients $C_{9}$ and $C_{10}$ defined in eq. (1.1), satisfying to $\delta C_{10}=-\delta C_{9}$.

Focussing on the flavour structure of $\mathcal{O}_{L L}^{(3)}$ and $\mathcal{O}_{L L}^{(5)}$, the two operators are invariant under the MFV flavour symmetry $\mathcal{G}_{Q} \times \mathcal{G}_{L}$, but can only describe flavour conserving observables which predict universality conservation in both the quark and lepton sectors. In order to describe a process with quark flavour change, it is then necessary to insert powers of the quark Yukawa spurion $\mathcal{Y}_{u}$. The dominant contributions would arise contracting the flavour indices of the quark bilinear with $\mathcal{Y}_{u} \mathcal{Y}_{u}^{\dagger}$ : once the spurions acquire their background values, the $b \rightarrow s$ transitions are weighted by the $V_{t b} V_{t s}^{*}$ factor appearing in eq. (1.1). Notice that, as $\left(Y_{u}\right)_{33}=y_{t} \approx 1$, an additional insertion of $\mathcal{Y}_{u} \mathcal{Y}_{u}^{\dagger}$ is not negligible and modifies the dominant contributions by $\left(1+y_{t}^{2}\right)$ factors. Further insertions of $\mathcal{Y}_{u} \mathcal{Y}_{u}^{\dagger}$ turn out to be unphysical, as they can be written as combinations of the linear and quadratic terms through the Cayley-Hamilton theorem. The complete spurion insertions in $\mathcal{O}_{L L}^{(3,5)}$ can then be written as $\zeta_{1} \mathcal{Y}_{u} \mathcal{Y}_{u}^{\dagger}+\zeta_{2}\left(\mathcal{Y}_{u} \mathcal{Y}_{u}^{\dagger}\right)^{2}$, with $\zeta_{1,2}$ arbitrary coefficients, reflecting the independence of each insertion: the net contribution to the operator is then given by $V_{t b} V_{t s}^{*}\left(\zeta_{1} y_{t}^{2}+\zeta_{2} y_{t}^{4}\right)$.

The anomalies in the angular observable $P_{5}^{\prime}$ of $B \rightarrow K^{*} \mu^{+} \mu^{-}$, in the ratios $R_{K}$ and $R_{K^{*}}$, and in the Branching Ratio of $B_{s} \rightarrow \phi \mu^{+} \mu^{-}$are linked to the possible violation of leptonic universality. NP contributions leading to these effects can be described in terms of insertions of spurion combinations transforming under 8 of SU $(3)_{\ell_{L}}$. The simplest structure is $\mathcal{Y}_{e} \mathcal{Y}_{e}^{\dagger}$ that, in the basis defined in eq. (2.12), is diagonal and therefore cannot lead to lepton flavour changing transitions. The phenomenological analysis associated to the insertion of this spurionic combination has been performed in ref. [211], where the focus was in understanding the consequences of having a setup where lepton universality is violated but lepton flavour is conserved. In ref. [211], the Abelian factors in eq. (2.8) are considered as active factors of the flavour symmetry and this leads to background values for $\mathcal{Y}_{e}$, whose largest eigenvalue is of order 1 . It should be noticed that strong constraints on this setup arise when considering radiative electroweak corrections as discussed in refs. [212, 213].

Focussing only on the non-Abelian factors, as in the tradicional MLFV, the largest entry of $Y_{e}$ is of the order of 0.01 , as can be seen from eq. (2.12). In this scenario, the insertion of $\mathcal{Y}_{e}$ is subdominant with respect to the insertion of the neutrino spurions: the most relevant are $\mathscr{g}_{\nu}^{\dagger} \mathscr{g}_{\nu}$ in the MFC, $\mathcal{Y}_{\nu} \mathcal{Y}_{\nu}^{\dagger}$ in the EFCI and $\mathcal{Y}_{N}^{\dagger} \mathcal{Y}_{N}$ in the EFCII. Once the spurions acquire background values, these contributions reduce to the $\Delta$ characteristic of each case. Similarly to what discussed above for $Y_{u}$, if the largest eigenvalue of $\Delta$ is of order 1 , then additional insertions of the neutrino spurions need to be taken into consideration. The specific contribution depends on the model considered and only a

\footnotetext{
${ }^{10}$ The complete effective Lagrangian that describes effects in $B$ physics can be found in ref. [209]. In particular, another operator, with respect to the reduced list in eq. (3.4), would contribute at tree level to $C_{9}, \bar{e}_{R} \gamma^{\mu} e_{R} \bar{q}_{L} \gamma_{\mu} q_{L}$ : this contribution is however negligible for the observables discussed here [210, 211], and then this operator is not considered in the present discussion.
} 
generic form $\sum_{n=0}^{2} \xi_{n} \Delta^{n}$ can be generically written, where $\xi_{n}$ are arbitrary Lagrangian coefficients, and where the sum is stopped at $n=2$ due to the Cayley-Hamilton theorem.

In ref. [154] the EFCI context has been considered and several processes have been studied, discussing the viability of this version of MLFV to consistently describe the $b \rightarrow s$ anomalies.

The aim of this section is to critically revisit the analysis of ref. [154], and to investigate the other two versions of MLFV. As already mentioned, EFCI will be disfavoured if the Dirac CP violation in the leptonic sector is confirmed, and therefore the viability of MFC and EFCII to describe the $b \rightarrow s$ anomalies, consistently with the other (un)observed flavour processes in the $B$ sector, becomes an interesting issue. Moreover, the results obtained in the previous section will be explicitly considered.

\subsection{B semi-leptonic decays}

In order to facilitate the comparison with ref. [154] similar assumptions will be taken. First of all, setting $C_{10}^{\mathrm{SM}}=-C_{9}^{\mathrm{SM}}$ and considering that the contributions from $\mathcal{O}_{L L}^{(3,5)}$ satisfy to $\delta C_{10}=-\delta C_{9}$, one can consider a single Wilson coefficient in eq. (1.1): for definiteness, $C_{9}$ will be retain in what follows. A second relevant assumption is on the matching between the effective operators of the high-energy Lagrangian defined at $\Lambda_{\mathrm{LFV}}$, eq. (3.4), and the low-energy phenomenological description in eq. (1.1): only the tree level relations will be considered in the following, while effects from loop-contributions and from the electroweak running will be neglected. The latter has been recently shown in refs. $[212,213]$ to lead to a rich phenomenology, especially in EWPO and $\tau$ sector.

Considering explicitly the contributions from $\mathcal{O}_{L L}^{(3,5)}$, and specifying the flavour indexes, one can write

$$
\delta C_{9, \ell \ell^{\prime}}=\frac{\pi}{\alpha_{\mathrm{em}}} \frac{v^{2}}{\Lambda_{\mathrm{LFV}}^{2}}\left(c_{L L, \ell \ell^{\prime}}^{(3)}+c_{L L, \ell \ell^{\prime}}^{(5)}\right)
$$

where $c_{L L, \ell \ell^{\prime}}^{(i)}$ can be written in a notation that makes explicit the dependence on the neutrino spurion background: ${ }^{11}$

$$
c_{L L, \ell \ell^{\prime}}^{(i)}=\left(\zeta_{1}^{(i)} y_{t}^{2}+\zeta_{2}^{(i)} y_{t}^{4}\right)\left(\xi_{0}^{(i)} \delta_{\ell \ell^{\prime}}+\xi_{1}^{(i)} \Delta_{\ell \ell^{\prime}}+\xi_{2}^{(i)} \Delta_{\ell \ell^{\prime}}\right)
$$

In order to explain lepton universality violation, the contributions proportional to $\xi_{1}^{(i)}$, $\xi_{2}^{(i)}$, etc. should be at least comparable with $\xi_{0}^{(i)}$. Consequently, this requires $\Delta_{\ell \ell} \sim 1$, and this allows to fix the scale of LNV: indeed, the bounds in eqs. (2.15), (2.25) and (2.30) become equalities,

$$
\begin{cases}\Lambda_{\mathrm{L}}=6 \times 10^{14} \mathrm{GeV}, & \text { for MFC } \\ \mu_{\mathrm{L}}=6 \times 10^{14} \mathrm{GeV}, & \text { for EFCI and EFCII } .\end{cases}
$$

\footnotetext{
${ }^{11}$ In ref. [154] a slightly different notation has been adopted, where

$$
c_{L L, \ell \ell^{\prime}}^{(i)}=\frac{\alpha_{\mathrm{em}}}{\pi} \frac{\Lambda_{\mathrm{LFV}}^{2}}{v^{2}}\left[\tilde{\xi}_{0}^{(i)} \delta_{\ell \ell^{\prime}}+\tilde{\xi}_{1}^{(i)} \Delta_{\ell \ell^{\prime}}+\tilde{\xi}_{2}^{(i)} \Delta_{\ell \ell^{\prime}}\right],
$$

with

$$
\tilde{\xi}_{j}^{(i)}=\frac{\pi}{\sqrt{2} \alpha_{\mathrm{em}} G_{F} \Lambda_{\mathrm{LFV}}^{2}}\left(\zeta_{1}^{(i)} y_{t}^{2}+\zeta_{2}^{(i)} y_{t}^{4}\right) \xi_{i}^{(i)} .
$$


The bounds from LFV purely leptonic processes discussed in the previous section allows to translate this result into specific values for the LFV scale: from the bounds on $\mu \rightarrow e$ conversion in nuclei, figure 1 , one obtains that

$$
\begin{cases}\Lambda_{\mathrm{LFV}}=4.4 \times 10^{5} \mathrm{GeV}, & \text { for MFC } \\ \Lambda_{\mathrm{LFV}}=2 \times 10^{5} \mathrm{GeV}, & \text { for EFCI } \\ \Lambda_{\mathrm{LFV}}=10^{5} \mathrm{GeV}, & \text { for EFCII }\end{cases}
$$

With these results at hand, the order of magnitude for $\delta C_{9}$ turns out to be

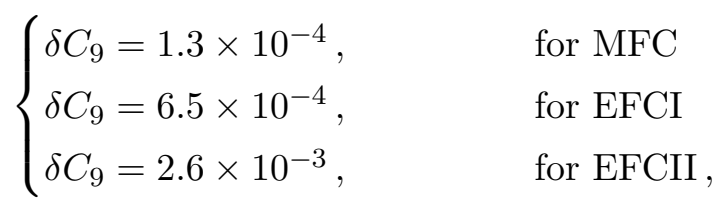

estimating only the pre-factors appearing in eq. (4.1). These values should now be compared with the ones in eq. (1.2), necessary to explain the anomalies in $b \rightarrow s$ decays: the version of MLFV that most contributes to the $C_{9}$ Wilson coefficient is EFCII, but its contributions are two order of magnitudes too small to explain the $B$ anomalies. It would be only by accident that the parameters of order 1 in eq. (4.4) combine together to compensate such suppression, but this would be an extremely tuned situation.

The only conclusion that can be deduced from this analysis is that all the three versions of MLFV cannot explain deviations from the SM predictions in the Wilson coefficient $C_{9}$ larger than a few per mil, once taking into consideration the bounds from leptonic radiative decays and conversion of muons in nuclei, contrary to what presented in previous literature.

If the anomalies in the $B$ sector will be confirmed, then it will be necessary to extend the MLFV context. Attempts in this directions have already appeared in the literature, although not motivated by the search for an explanation of the $b \rightarrow s$ decay anomalies. The flavour symmetry of the M(L)FV is a continuous global symmetry and therefore, once promoting the spurions to dynamical fields, its spontaneous breaking leads to the arising of Goldstone bosons. Although it would be possible to provide masses for these new states, this would require an explicit breaking of the flavour symmetry. An alternative is to gauge the symmetry [79-83, 88]: the would-be-Goldstone bosons would be eaten by flavour gauge bosons that enrich the spectrum. In recent papers [214, 215], a specific gauge boson arising from the chosen gauged flavour symmetry has the specific couplings to explain the $b \rightarrow s$ anomalies here mentioned.

\section{Conclusions}

The MFV is a framework to describe fermion masses and mixings and to provide at the same time a sort of flavour protection from beyond the Standard Model contributions to flavour processes. The lack of knowledge of the neutrino mass origin reflects in a larger freedom when implementing the MFV ansatz in the lepton sector: three distinct versions of the MLFV have been proposed in the literature. 
In the present paper, an update of the phenomenological analyses on these setups is presented considering the most recent fit on the neutrino oscillation data. The recent indication of $\mathrm{CP}$ violation in the leptonic sector, if confirmed, will disfavour the very popular MLFV version [62] called here EFCI, where right-handed neutrinos are assumed to be degenerate at tree level and the flavour symmetry is $\mathrm{SU}(3)_{\ell_{L}} \times \mathrm{SU}(3)_{e_{R}} \times \mathrm{SO}(3)_{N_{R}} \times C P$.

The study of the predictions within these frameworks for flavour changing processes has been presented, focussing on leptonic radiative rare decays and muon conversion in nuclei, which provide the stringent bounds. A strategy to disentangle between the different MLFV possibilities has been described: in particular, the next future experiments searching for $\mu \rightarrow e \gamma$ and $\mu \rightarrow e$ conversion in aluminium could have the power to pinpoint the scenario described here as EFCII [65], characterised by the flavour symmetry $\mathrm{SU}(3)_{\ell_{L}+N_{R}} \times \mathrm{SU}(3)_{e_{R}}$, if the neutrino mass spectrum is normal ordered.

An interesting question is whether the present anomalies in the semi-leptonic $B$-meson decays can find an explanation within the $\mathrm{M}(\mathrm{L}) \mathrm{FV}$ context. Contrary to what claimed in the literature, such an explanation would require a scale of New Physics that turns out to be excluded once considering purely leptonic processes, the limits on the rate of muon conversion in nuclei being the most constraining. These anomalies could find a solution extending/modifying the M(L)FV setup, for example, by gauging the flavour symmetry.

\section{Acknowledgments}

L.M. thanks the department of Physics and Astronomy of the Università degli Studi di Padova for the hospitality during the writing up of this paper and Paride Paradisi for useful comments on this project and for all the enjoyable discussions during this visit. D.N.D. thanks the Department of Physics of the University of Virginia for the hospitality and P.Q. Hung for the exciting discussions and kind helps.

D.N.D. acknowledges partial support by the Vietnam National Foundation for Science and Technology Development (NAFOSTED) under the grant 103.01-2014.89, and by the Vietnam Education Foundation (VEF) for the scholarship to work at the Department of Physics of the University of Virginia. L.M. and S.T.P. acknowledge partial financial support by the European Union's Horizon 2020 research and innovation programme under the Marie Sklodowska-Curie grant agreements No 690575 and No 674896. The work of L.M. was supported in part also by "Spanish Agencia Estatal de Investigación" (AEI) and the EU "Fondo Europeo de Desarrollo Regional" (FEDER) through the project FPA201678645-P, and by the Spanish MINECO through the Centro de excelencia Severo Ochoa Program under grant SEV-2012-0249 and by the Spanish MINECO through the "Ramón y Cajal" programme (RYC-2015-17173). The work of S.T.P. was supported in part by the INFN program on Theoretical Astroparticle Physics (TASP) and by the World Premier International Research Center Initiative (WPI Initiative), MEXT, Japan. 
Open Access. This article is distributed under the terms of the Creative Commons Attribution License (CC-BY 4.0), which permits any use, distribution and reproduction in any medium, provided the original author(s) and source are credited.

\section{References}

[1] T2K collaboration, K. Abe et al., Indication of Electron Neutrino Appearance from an Accelerator-produced Off-axis Muon Neutrino Beam, Phys. Rev. Lett. 107 (2011) 041801 [arXiv:1106.2822] [INSPIRE].

[2] MINOS collaboration, P. Adamson et al., Improved search for muon-neutrino to electron-neutrino oscillations in MINOS, Phys. Rev. Lett. 107 (2011) 181802 [arXiv: 1108.0015] [INSPIRE].

[3] Double CHOOZ collaboration, Y. Abe et al., Indication of Reactor $\bar{\nu}_{e}$ Disappearance in the Double CHOOZ Experiment, Phys. Rev. Lett. 108 (2012) 131801 [arXiv:1112.6353] [INSPIRE].

[4] DAYA BAY collaboration, F.P. An et al., Observation of electron-antineutrino disappearance at Daya Bay, Phys. Rev. Lett. 108 (2012) 171803 [arXiv:1203.1669] [INSPIRE].

[5] RENO collaboration, J.K. Ahn et al., Observation of Reactor Electron Antineutrino Disappearance in the RENO Experiment, Phys. Rev. Lett. 108 (2012) 191802 [arXiv:1204.0626] [INSPIRE].

[6] T. Fukuyama and H. Nishiura, Mass matrix of Majorana neutrinos, hep-ph/9702253 [INSPIRE].

[7] G. Altarelli and F. Feruglio, Models of neutrino masses from oscillations with maximal mixing, JHEP 11 (1998) 021 [hep-ph/9809596] [INSPIRE].

[8] P.F. Harrison, D.H. Perkins and W.G. Scott, Tri-bimaximal mixing and the neutrino oscillation data, Phys. Lett. B 530 (2002) 167 [hep-ph/0202074] [INSPIRE].

[9] P.F. Harrison and W.G. Scott, Symmetries and generalizations of tri- bimaximal neutrino mixing, Phys. Lett. B 535 (2002) 163 [hep-ph/0203209] [INSPIRE].

[10] Z.-z. Xing, Nearly tri bimaximal neutrino mixing and CP-violation, Phys. Lett. B 533 (2002) 85 [hep-ph/0204049] [INSPIRE].

[11] E. Ma and G. Rajasekaran, Softly broken $A_{4}$ symmetry for nearly degenerate neutrino masses, Phys. Rev. D 64 (2001) 113012 [hep-ph/0106291] [INSPIRE].

[12] K.S. Babu, E. Ma and J.W.F. Valle, Underlying $A_{4}$ symmetry for the neutrino mass matrix and the quark mixing matrix, Phys. Lett. B 552 (2003) 207 [hep-ph/0206292] [INSPIRE].

[13] G. Altarelli and F. Feruglio, Tri-bimaximal neutrino mixing from discrete symmetry in extra dimensions, Nucl. Phys. B 720 (2005) 64 [hep-ph/0504165] [INSPIRE].

[14] G. Altarelli and F. Feruglio, Tri-bimaximal neutrino mixing, $A_{4}$ and the modular symmetry, Nucl. Phys. B 741 (2006) 215 [hep-ph/0512103] [INSPIRE].

[15] G. Altarelli, F. Feruglio and Y. Lin, Tri-bimaximal neutrino mixing from orbifolding, Nucl. Phys. B 775 (2007) 31 [hep-ph/0610165] [INSPIRE].

[16] I. de Medeiros Varzielas, S.F. King and G.G. Ross, Neutrino tri-bi-maximal mixing from a non-Abelian discrete family symmetry, Phys. Lett. B 648 (2007) 201 [hep-ph/0607045] [INSPIRE]. 
[17] F. Feruglio, C. Hagedorn, Y. Lin and L. Merlo, Tri-bimaximal Neutrino Mixing and Quark Masses from a Discrete Flavour Symmetry, Nucl. Phys. B 775 (2007) 120 [Erratum ibid. B 836 (2010) 127] [hep-ph/0702194] [INSPIRE].

[18] F. Bazzocchi, L. Merlo and S. Morisi, Fermion Masses and Mixings in a $S_{4}$-based Model, Nucl. Phys. B 816 (2009) 204 [arXiv:0901.2086] [INSPIRE].

[19] F. Bazzocchi, L. Merlo and S. Morisi, Phenomenological Consequences of See-Saw in $S_{4}$ Based Models, Phys. Rev. D 80 (2009) 053003 [arXiv: 0902. 2849] [InSPIRE].

[20] S.T. Petcov, On PseudoDirac Neutrinos, Neutrino Oscillations and Neutrinoless Double beta Decay, Phys. Lett. B 110 (1982) 245 [InSPIRE].

[21] F. Vissani, A Study of the scenario with nearly degenerate Majorana neutrinos, hep-ph/9708483 [INSPIRE].

[22] V.D. Barger, S. Pakvasa, T.J. Weiler and K. Whisnant, Bimaximal mixing of three neutrinos, Phys. Lett. B 437 (1998) 107 [hep-ph/9806387] [INSPIRE].

[23] Y. Kajiyama, M. Raidal and A. Strumia, The golden ratio prediction for the solar neutrino mixing, Phys. Rev. D 76 (2007) 117301 [arXiv:0705.4559] [INSPIRE].

[24] W. Rodejohann, Unified Parametrization for Quark and Lepton Mixing Angles, Phys. Lett. B 671 (2009) 267 [arXiv:0810.5239] [INSPIRE].

[25] S.F. King and C. Luhn, Trimaximal neutrino mixing from vacuum alignment in A4 and S4 models, JHEP 09 (2011) 042 [arXiv:1107.5332] [INSPIRE].

[26] P.H. Frampton, S.T. Petcov and W. Rodejohann, On deviations from bimaximal neutrino mixing, Nucl. Phys. B 687 (2004) 31 [hep-ph/0401206] [INSPIRE].

[27] A. Romanino, Charged lepton contributions to the solar neutrino mixing and $\theta_{13}$, Phys. Rev. D 70 (2004) 013003 [hep-ph/0402258] [INSPIRE].

[28] G. Altarelli, F. Feruglio and I. Masina, Can neutrino mixings arise from the charged lepton sector?, Nucl. Phys. B 689 (2004) 157 [hep-ph/0402155] [inSPIRE].

[29] K.A. Hochmuth, S.T. Petcov and W. Rodejohann, $U_{P M N S}=U_{\ell}^{\dagger} U_{\nu}$, Phys. Lett. B 654 (2007) 177 [arXiv: 0706.2975] [INSPIRE].

[30] S.T. Petcov and A.Yu. Smirnov, Neutrinoless double beta decay and the solar neutrino problem, Phys. Lett. B 322 (1994) 109 [hep-ph/9311204] [INSPIRE].

[31] H. Minakata and A.Yu. Smirnov, Neutrino mixing and quark-lepton complementarity, Phys. Rev. D 70 (2004) 073009 [hep-ph/0405088] [INSPIRE].

[32] G. Altarelli, F. Feruglio and L. Merlo, Revisiting Bimaximal Neutrino Mixing in a Model with $S_{4}$ Discrete Symmetry, JHEP 05 (2009) 020 [arXiv:0903.1940] [INSPIRE].

[33] R. de Adelhart Toorop, F. Bazzocchi and L. Merlo, The Interplay Between GUT and Flavour Symmetries in a Pati-Salam $\times S_{4}$ Model, JHEP 08 (2010) 001 [arXiv:1003.4502] [INSPIRE].

[34] D. Meloni, Bimaximal mixing and large $\theta_{13}$ in a SUSY SU(5) model based on $S_{4}$, JHEP 10 (2011) 010 [arXiv: 1107.0221] [INSPIRE].

[35] G. Altarelli and F. Feruglio, Discrete Flavor Symmetries and Models of Neutrino Mixing, Rev. Mod. Phys. 82 (2010) 2701 [arXiv: 1002.0211] [InSPIRE].

[36] W. Grimus and P.O. Ludl, Finite flavour groups of fermions, J. Phys. A 45 (2012) 233001 [arXiv:1110.6376] [INSPIRE]. 
[37] G. Altarelli, F. Feruglio and L. Merlo, Tri-Bimaximal Neutrino Mixing and Discrete Flavour Symmetries, Fortsch. Phys. 61 (2013) 507 [arXiv: 1205.5133] [INSPIRE].

[38] F. Bazzocchi and L. Merlo, Neutrino Mixings and the S4 Discrete Flavour Symmetry, Fortsch. Phys. 61 (2013) 571 [arXiv:1205.5135] [INSPIRE].

[39] S.F. King and C. Luhn, Neutrino Mass and Mixing with Discrete Symmetry, Rept. Prog. Phys. 76 (2013) 056201 [arXiv: 1301.1340] [INSPIRE].

[40] S.F. King, Models of Neutrino Mass, Mixing and CP-violation, J. Phys. G 42 (2015) 123001 [arXiv: 1510.02091] [INSPIRE].

[41] F. Capozzi, E. Lisi, A. Marrone, D. Montanino and A. Palazzo, Neutrino masses and

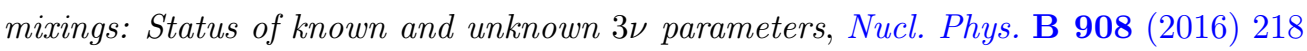
[arXiv: 1601 .07777] [INSPIRE].

[42] I. Esteban, M.C. Gonzalez-Garcia, M. Maltoni, I. Martínez-Soler and T. Schwetz, Updated fit to three neutrino mixing: exploring the accelerator-reactor complementarity, JHEP 01 (2017) 087 [arXiv: 1611.01514] [INSPIRE].

[43] F. Capozzi, E. Di Valentino, E. Lisi, A. Marrone, A. Melchiorri and A. Palazzo, Global constraints on absolute neutrino masses and their ordering, Phys. Rev. D 95 (2017) 096014 [arXiv: 1703.04471] [INSPIRE].

[44] E. Ma and D. Wegman, Nonzero $\theta_{13}$ for neutrino mixing in the context of $A_{4}$ symmetry, Phys. Rev. Lett. 107 (2011) 061803 [arXiv:1106.4269] [INSPIRE].

[45] S.F. King and C. Luhn, $A_{4}$ models of tri-bimaximal-reactor mixing, JHEP 03 (2012) 036 [arXiv:1112.1959] [INSPIRE].

[46] Y. Lin, Tri-bimaximal Neutrino Mixing from $A_{4}$ and $\theta_{13} \sim \theta_{C}$, Nucl. Phys. B 824 (2010) 95 [arXiv: 0905. 3534] [INSPIRE].

[47] G. Altarelli and D. Meloni, A Simplest A4 Model for Tri-Bimaximal Neutrino Mixing, J. Phys. G 36 (2009) 085005 [arXiv:0905.0620] [INSPIRE].

[48] I. de Medeiros Varzielas and L. Merlo, Ultraviolet Completion of Flavour Models, JHEP 02 (2011) 062 [arXiv: 1011.6662] [INSPIRE].

[49] G. Altarelli, F. Feruglio, L. Merlo and E. Stamou, Discrete Flavour Groups, $\theta_{13}$ and Lepton Flavour Violation, JHEP 08 (2012) 021 [arXiv: 1205.4670] [INSPIRE].

[50] R. de Adelhart Toorop, F. Feruglio and C. Hagedorn, Discrete Flavour Symmetries in Light of T2K, Phys. Lett. B 703 (2011) 447 [arXiv:1107.3486] [InSPIRE].

[51] R. de Adelhart Toorop, F. Feruglio and C. Hagedorn, Finite Modular Groups and Lepton Mixing, Nucl. Phys. B 858 (2012) 437 [arXiv:1112.1340] [INSPIRE].

[52] C.D. Froggatt and H.B. Nielsen, Hierarchy of Quark Masses, Cabibbo Angles and CP-violation, Nucl. Phys. B 147 (1979) 277 [inSPIRE].

[53] G. Altarelli, F. Feruglio and I. Masina, From minimal to realistic supersymmetric SU(5) grand unification, JHEP 11 (2000) 040 [hep-ph/0007254] [INSPIRE].

[54] G. Altarelli, F. Feruglio and I. Masina, Models of neutrino masses: Anarchy versus hierarchy, JHEP 01 (2003) 035 [hep-ph/0210342] [INSPIRE].

[55] W. Buchmüller, V. Domcke and K. Schmitz, Predicting $\theta_{13}$ and the Neutrino Mass Scale from Quark Lepton Mass Hierarchies, JHEP 03 (2012) 008 [arXiv:1111.3872] [INSPIRE].

[56] G. Altarelli, F. Feruglio, I. Masina and L. Merlo, Repressing Anarchy in Neutrino Mass Textures, JHEP 11 (2012) 139 [arXiv: 1207.0587] [INSPIRE]. 
[57] J. Bergstrom, D. Meloni and L. Merlo, Bayesian comparison of U(1) lepton flavor models, Phys. Rev. D 89 (2014) 093021 [arXiv:1403.4528] [InSPIRE].

[58] S.F. King and G.G. Ross, Fermion masses and mixing angles from SU(3) family symmetry, Phys. Lett. B 520 (2001) 243 [hep-ph/0108112] [INSPIRE].

[59] S.F. King and G.G. Ross, Fermion masses and mixing angles from SU(3) family symmetry and unification, Phys. Lett. B 574 (2003) 239 [hep-ph/0307190] [INSPIRE].

[60] R.S. Chivukula and H. Georgi, Composite Technicolor Standard Model, Phys. Lett. B 188 (1987) 99 [INSPIRE].

[61] G. D'Ambrosio, G.F. Giudice, G. Isidori and A. Strumia, Minimal flavor violation: An effective field theory approach, Nucl. Phys. B 645 (2002) 155 [hep-ph/0207036] [InSPIRE].

[62] V. Cirigliano, B. Grinstein, G. Isidori and M.B. Wise, Minimal flavor violation in the lepton sector, Nucl. Phys. B 728 (2005) 121 [hep-ph/0507001] [INSPIRE].

[63] S. Davidson and F. Palorini, Various definitions of Minimal Flavour Violation for Leptons, Phys. Lett. B 642 (2006) 72 [hep-ph/0607329] [INSPIRE].

[64] M.B. Gavela, T. Hambye, D. Hernandez and P. Hernández, Minimal Flavour Seesaw Models, JHEP 09 (2009) 038 [arXiv:0906.1461] [INSPIRE].

[65] R. Alonso, G. Isidori, L. Merlo, L.A. Muñoz and E. Nardi, Minimal flavour violation extensions of the seesaw, JHEP 06 (2011) 037 [arXiv:1103.5461] [INSPIRE].

[66] A. Anselm and Z. Berezhiani, Weak mixing angles as dynamical degrees of freedom, Nucl. Phys. B 484 (1997) 97 [hep-ph/9605400] [INSPIRE].

[67] R. Barbieri, L.J. Hall, G.L. Kane and G.G. Ross, Nearly degenerate neutrinos and broken flavor symmetry, hep-ph/9901228 [INSPIRE].

[68] Z. Berezhiani and A. Rossi, Flavor structure, flavor symmetry and supersymmetry, Nucl. Phys. Proc. Suppl. 101 (2001) 410 [hep-ph/0107054] [INSPIRE].

[69] T. Feldmann, M. Jung and T. Mannel, Sequential Flavour Symmetry Breaking, Phys. Rev. D 80 (2009) 033003 [arXiv:0906.1523] [INSPIRE].

[70] R. Alonso, M.B. Gavela, L. Merlo and S. Rigolin, On the scalar potential of minimal flavour violation, JHEP 07 (2011) 012 [arXiv:1103.2915] [InSPIRE].

[71] E. Nardi, Naturally large Yukawa hierarchies, Phys. Rev. D 84 (2011) 036008 [arXiv: 1105.1770] [INSPIRE].

[72] R. Alonso, M.B. Gavela, D. Hernandez and L. Merlo, On the Potential of Leptonic Minimal Flavour Violation, Phys. Lett. B 715 (2012) 194 [arXiv:1206.3167] [INSPIRE].

[73] R. Alonso, M.B. Gavela, D. Hernández, L. Merlo and S. Rigolin, Leptonic Dynamical Yukawa Couplings, JHEP 08 (2013) 069 [arXiv: 1306.5922] [INSPIRE].

[74] R. Alonso, M.B. Gavela, G. Isidori and L. Maiani, Neutrino Mixing and Masses from a Minimum Principle, JHEP 11 (2013) 187 [arXiv:1306.5927] [INSPIRE].

[75] C.S. Fong and E. Nardi, Quark masses, mixings and CP-violation from spontaneous breaking of flavor $\mathrm{SU}(3)^{3}$, Phys. Rev. D 89 (2014) 036008 [arXiv:1307.4412] [INSPIRE].

[76] V. Cirigliano and B. Grinstein, Phenomenology of minimal lepton flavor violation, Nucl. Phys. B 752 (2006) 18 [hep-ph/0601111] [INSPIRE].

[77] B. Grinstein, V. Cirigliano, G. Isidori and M.B. Wise, Grand Unification and the Principle of Minimal Flavor Violation, Nucl. Phys. B 763 (2007) 35 [hep-ph/0608123] [INSPIRE]. 
[78] P. Paradisi and D.M. Straub, The SUSY CP Problem and the MFV Principle, Phys. Lett. B 684 (2010) 147 [arXiv:0906.4551] [InSPIRE].

[79] B. Grinstein, M. Redi and G. Villadoro, Low Scale Flavor Gauge Symmetries, JHEP 11 (2010) 067 [arXiv: 1009.2049] [InSPIRE].

[80] T. Feldmann, See-Saw Masses for Quarks and Leptons in SU(5), JHEP 04 (2011) 043 [arXiv: 1010.2116] [INSPIRE].

[81] D. Guadagnoli, R.N. Mohapatra and I. Sung, Gauged Flavor Group with Left-Right Symmetry, JHEP 04 (2011) 093 [arXiv: 1103.4170] [INSPIRE].

[82] A.J. Buras, L. Merlo and E. Stamou, The Impact of Flavour Changing Neutral Gauge Bosons on $\bar{B} \rightarrow X_{s} \gamma$, JHEP 08 (2011) 124 [arXiv:1105.5146] [INSPIRE].

[83] A.J. Buras, M.V. Carlucci, L. Merlo and E. Stamou, Phenomenology of a Gauged SU(3) ${ }^{3}$ Flavour Model, JHEP 03 (2012) 088 [arXiv:1112.4477] [INSPIRE].

[84] R. Alonso, M.B. Gavela, L. Merlo, S. Rigolin and J. Yepes, Minimal Flavour Violation with Strong Higgs Dynamics, JHEP 06 (2012) 076 [arXiv:1201.1511] [INSPIRE].

[85] R. Alonso, M.B. Gavela, L. Merlo, S. Rigolin and J. Yepes, Flavor with a light dynamical "Higgs particle", Phys. Rev. D 87 (2013) 055019 [arXiv:1212.3307] [INSPIRE].

[86] L. Lopez-Honorez and L. Merlo, Dark matter within the minimal flavour violation ansatz, Phys. Lett. B 722 (2013) 135 [arXiv: 1303.1087] [INSPIRE].

[87] R. Barbieri, D. Buttazzo, F. Sala and D.M. Straub, Flavour physics and flavour symmetries after the first LHC phase, JHEP 05 (2014) 105 [arXiv: 1402.6677] [INSPIRE].

[88] R. Alonso, E. Fernandez Martínez, M.B. Gavela, B. Grinstein, L. Merlo and P. Quilez, Gauged Lepton Flavour, JHEP 12 (2016) 119 [arXiv:1609.05902] [INSPIRE].

[89] A. Crivellin, J. Fuentes-Martin, A. Greljo and G. Isidori, Lepton Flavor Non-Universality in B decays from Dynamical Yukawas, Phys. Lett. B 766 (2017) 77 [arXiv:1611.02703] [INSPIRE].

[90] D.V. Forero, M. Tortola and J.W.F. Valle, Neutrino oscillations refitted, Phys. Rev. D 90 (2014) 093006 [arXiv:1405.7540] [inSPIRE].

[91] M. Blennow, P. Coloma and E. Fernandez-Martínez, Reassessing the sensitivity to leptonic CP-violation, JHEP 03 (2015) 005 [arXiv: 1407.3274] [INSPIRE].

[92] F. Capozzi, G.L. Fogli, E. Lisi, A. Marrone, D. Montanino and A. Palazzo, Status of three-neutrino oscillation parameters, circa 2013, Phys. Rev. D 89 (2014) 093018 [arXiv:1312.2878] [INSPIRE].

[93] Particle Data Group collaboration, C. Patrignani et al., Review of Particle Physics, Chin. Phys. C 40 (2016) 100001 [inSPIRE].

[94] F. Feruglio, C. Hagedorn and R. Ziegler, Lepton Mixing Parameters from Discrete and CP Symmetries, JHEP 07 (2013) 027 [arXiv:1211.5560] [INSPIRE].

[95] M. Holthausen, M. Lindner and M.A. Schmidt, CP and Discrete Flavour Symmetries, JHEP 04 (2013) 122 [arXiv:1211.6953] [INSPIRE].

[96] F. Feruglio, C. Hagedorn and R. Ziegler, A realistic pattern of lepton mixing and masses from $S_{4}$ and CP, Eur. Phys. J. C 74 (2014) 2753 [arXiv:1303.7178] [InSPIRE].

[97] I. Girardi, A. Meroni, S.T. Petcov and M. Spinrath, Generalised geometrical CP-violation in a $T^{\prime}$ lepton flavour model, JHEP 02 (2014) 050 [arXiv: 1312.1966] [INSPIRE]. 
[98] G.C. Branco, I. de Medeiros Varzielas and S.F. King, Invariant approach to $\mathcal{C P}$ in unbroken $\Delta(27)$, Nucl. Phys. B 899 (2015) 14 [arXiv:1505.06165] [InSPIRE].

[99] G.-J. Ding and S.F. King, Generalized CP and $\Delta\left(3 n^{2}\right)$ Family Symmetry for Semi-Direct Predictions of the PMNS Matrix, Phys. Rev. D 93 (2016) 025013 [arXiv:1510.03188] [INSPIRE].

[100] I. de Medeiros Varzielas, S.F. King, C. Luhn and T. Neder, CP-odd invariants for multi-Higgs models: applications with discrete symmetry, Phys. Rev. D 94 (2016) 056007 [arXiv: 1603.06942] [INSPIRE].

[101] Y. Shimizu, M. Tanimoto and K. Yamamoto, Predicting CP-violation in Deviation from Tri-bimaximal mixing of Neutrinos, Mod. Phys. Lett. A 30 (2015) 1550002 [arXiv:1405.1521] [INSPIRE].

[102] S.T. Petcov, Predicting the values of the leptonic CP-violation phases in theories with discrete flavour symmetries, Nucl. Phys. B 892 (2015) 400 [arXiv:1405.6006] [INSPIRE].

[103] I. Girardi, S.T. Petcov and A.V. Titov, Predictions for the Leptonic Dirac CP-violation Phase: a Systematic Phenomenological Analysis, Eur. Phys. J. C 75 (2015) 345 [arXiv: 1504.00658] [INSPIRE].

[104] I. Girardi, S.T. Petcov, A.J. Stuart and A.V. Titov, Leptonic Dirac CP-violation Predictions from Residual Discrete Symmetries, Nucl. Phys. B 902 (2016) 1 [arXiv: 1509. 02502] [INSPIRE].

[105] S.F. King, A. Merle and A.J. Stuart, The Power of Neutrino Mass Sum Rules for Neutrinoless Double Beta Decay Experiments, JHEP 12 (2013) 005 [arXiv:1307.2901] [INSPIRE].

[106] P. Ballett, S.F. King, C. Luhn, S. Pascoli and M.A. Schmidt, Testing atmospheric mixing sum rules at precision neutrino facilities, Phys. Rev. D 89 (2014) 016016 [arXiv: 1308.4314] [INSPIRE].

[107] P. Ballett, S.F. King, C. Luhn, S. Pascoli and M.A. Schmidt, Testing solar lepton mixing sum rules in neutrino oscillation experiments, JHEP 12 (2014) 122 [arXiv:1410.7573] [INSPIRE].

[108] I. Girardi, S.T. Petcov and A.V. Titov, Determining the Dirac CP-violation Phase in the Neutrino Mixing Matrix from Sum Rules, Nucl. Phys. B 894 (2015) 733 [arXiv: 1410.8056] [INSPIRE].

[109] J. Gehrlein, A. Merle and M. Spinrath, Predictivity of Neutrino Mass Sum Rules, Phys. Rev. D 94 (2016) 093003 [arXiv: 1606. 04965] [INSPIRE].

[110] R. Barbieri, G. Isidori, J. Jones-Perez, P. Lodone and D.M. Straub, U(2) and Minimal Flavour Violation in Supersymmetry, Eur. Phys. J. C 71 (2011) 1725 [arXiv:1105.2296] [INSPIRE].

[111] R. Barbieri, P. Campli, G. Isidori, F. Sala and D.M. Straub, B-decay CP-asymmetries in SUSY with a U(2) flavour symmetry, Eur. Phys. J. C 71 (2011) 1812 [arXiv:1108.5125] [INSPIRE].

[112] R. Barbieri, D. Buttazzo, F. Sala and D.M. Straub, Flavour physics from an approximate $\mathrm{U}(2)^{3}$ symmetry, JHEP 07 (2012) 181 [arXiv: 1203.4218] [INSPIRE].

[113] R. Barbieri, D. Buttazzo, F. Sala and D.M. Straub, Less Minimal Flavour Violation, JHEP 10 (2012) 040 [arXiv: 1206.1327] [INSPIRE]. 
[114] R. Barbieri, G. Isidori, A. Pattori and F. Senia, Anomalies in B-decays and U(2) flavour symmetry, Eur. Phys. J. C 76 (2016) 67 [arXiv:1512.01560] [INSPIRE].

[115] M. Bordone, G. Isidori and S. Trifinopoulos, Semi-leptonic B-physics anomalies: a general EFT analysis within $\mathrm{U}(2)^{n}$ flavor symmetry, arXiv:1702.07238 [INSPIRE].

[116] F. Feruglio, C. Hagedorn, Y. Lin and L. Merlo, Lepton Flavour Violation in Models with $A_{4}$ Flavour Symmetry, Nucl. Phys. B 809 (2009) 218 [arXiv:0807.3160] [INSPIRE].

[117] H. Ishimori, T. Kobayashi, H. Okada, Y. Shimizu and M. Tanimoto, Lepton Flavor Model from Delta(54) Symmetry, JHEP 04 (2009) 011 [arXiv: 0811.4683] [INSPIRE].

[118] F. Feruglio, C. Hagedorn, Y. Lin and L. Merlo, Lepton Flavour Violation in a Supersymmetric Model with $A_{4}$ Flavour Symmetry, Nucl. Phys. B 832 (2010) 251 [arXiv:0911.3874] [INSPIRE].

[119] F. Feruglio, C. Hagedorn and L. Merlo, Vacuum Alignment in SUSY A4 Models, JHEP 03 (2010) 084 [arXiv: 0910.4058] [InSPIRE].

[120] R. de Adelhart Toorop, F. Bazzocchi, L. Merlo and A. Paris, Constraining Flavour Symmetries At The EW Scale I: The A4 Higgs Potential, JHEP 03 (2011) 035 [Erratum ibid. 01 (2013) 098] [arXiv: 1012.1791] [INSPIRE].

[121] R. de Adelhart Toorop, F. Bazzocchi, L. Merlo and A. Paris, Constraining Flavour Symmetries At The EW Scale II: The Fermion Processes, JHEP 03 (2011) 040 [arXiv: 1012.2091] [INSPIRE].

[122] H. Ishimori and M. Tanimoto, Slepton Mass Matrices, $\mu \rightarrow e \gamma$ Decay and EDM in SUSY S4 Flavor Model, Prog. Theor. Phys. 125 (2011) 653 [arXiv:1012.2232] [InSPIRE].

[123] L. Merlo, S. Rigolin and B. Zaldivar, Flavour violation in a supersymmetric $T^{\prime}$ model, JHEP 11 (2011) 047 [arXiv:1108.1795] [InSPIRE].

[124] LHCb collaboration, Measurement of Form-Factor-Independent Observables in the Decay $B^{0} \rightarrow K^{* 0} \mu^{+} \mu^{-}$, Phys. Rev. Lett. 111 (2013) 191801 [arXiv:1308.1707] [InSPIRE].

[125] LHCb collaboration, Angular analysis of the $B^{0} \rightarrow K^{* 0} \mu^{+} \mu^{-}$decay using $3 \mathrm{fb}^{-1}$ of integrated luminosity, JHEP 02 (2016) 104 [arXiv:1512.04442] [INSPIRE].

[126] Belle collaboration, A. Abdesselam et al., Angular analysis of $B^{0} \rightarrow K^{*}(892)^{0} \ell^{+} \ell^{-}$, arXiv: 1604.04042 [INSPIRE].

[127] LHCb collaboration, Angular analysis and differential branching fraction of the decay $B_{s}^{0} \rightarrow \phi \mu^{+} \mu^{-}$, JHEP 09 (2015) 179 [arXiv: 1506.08777] [INSPIRE].

[128] S. Fajfer, J.F. Kamenik and I. Nisandzic, On the $B \rightarrow D^{*} \tau \bar{\nu}_{\tau}$ Sensitivity to New Physics, Phys. Rev. D 85 (2012) 094025 [arXiv:1203.2654] [INSPIRE].

[129] BaBAR collaboration, J.P. Lees et al., Measurement of an Excess of $\bar{B} \rightarrow D^{(*)} \tau^{-} \bar{\nu}_{\tau}$ Decays and Implications for Charged Higgs Bosons, Phys. Rev. D 88 (2013) 072012 [arXiv: 1303.0571] [INSPIRE].

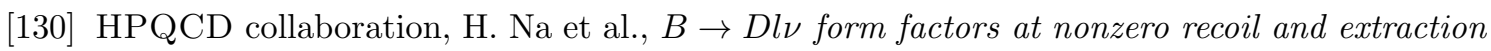
of $\left|V_{c b}\right|$, Phys. Rev. D 92 (2015) 054510 [arXiv: 1505.03925] [INSPIRE].

[131] LHCb collaboration, Measurement of the ratio of branching fractions $\mathcal{B}\left(\bar{B}^{0} \rightarrow D^{*+} \tau^{-} \bar{\nu}_{\tau}\right) / \mathcal{B}\left(\bar{B}^{0} \rightarrow D^{*+} \mu^{-} \bar{\nu}_{\mu}\right)$, Phys. Rev. Lett. 115 (2015) 111803 [arXiv: 1506.08614] [INSPIRE].

[132] BELLE collaboration, M. Huschle et al., Measurement of the branching ratio of $\bar{B} \rightarrow D^{(*)} \tau^{-} \bar{\nu}_{\tau}$ relative to $\bar{B} \rightarrow D^{(*)} \ell^{-} \bar{\nu}_{\ell}$ decays with hadronic tagging at Belle, Phys. Rev. D 92 (2015) 072014 [arXiv:1507.03233] [INSPIRE]. 
[133] LHCb collaboration, Test of lepton universality using $B^{+} \rightarrow K^{+} \ell^{+} \ell^{-}$decays, Phys. Rev. Lett. 113 (2014) 151601 [arXiv: 1406.6482] [INSPIRE].

[134] S. Bifani, Search for new physics with $b \rightarrow s \ell^{+} \ell^{-}$decays at LHCb, CERN Seminar, 18 April 2017.

[135] J. Lyon and R. Zwicky, Resonances gone topsy turvy - the charm of QCD or new physics in $b \rightarrow s \ell^{+} \ell^{-}$?, arXiv:1406.0566 [INSPIRE].

[136] S. Descotes-Genon, L. Hofer, J. Matias and J. Virto, On the impact of power corrections in the prediction of $B \rightarrow K^{*} \mu^{+} \mu^{-}$observables, JHEP 12 (2014) 125 [arXiv:1407.8526] [INSPIRE].

[137] S. Jäger and J. Martin Camalich, Reassessing the discovery potential of the $B \rightarrow K^{*} \ell^{+} \ell^{-}$ decays in the large-recoil region: SM challenges and BSM opportunities, Phys. Rev. D 93 (2016) 014028 [arXiv: 1412.3183] [INSPIRE].

[138] M. Ciuchini et al., $B \rightarrow K^{*} \ell^{+} \ell^{-}$decays at large recoil in the Standard Model: a theoretical reappraisal, JHEP 06 (2016) 116 [arXiv:1512.07157] [INSPIRE].

[139] B. Capdevila, S. Descotes-Genon, L. Hofer and J. Matias, Hadronic uncertainties in $B \rightarrow K^{*} \mu^{+} \mu^{-}$: a state-of-the-art analysis, JHEP 04 (2017) 016 [arXiv:1701.08672] [INSPIRE].

[140] V.G. Chobanova, T. Hurth, F. Mahmoudi, D. Martínez Santos and S. Neshatpour, Large hadronic power corrections or new physics in the rare decay $B \rightarrow K^{*} \mu^{+} \mu^{-}$?, JHEP 07 (2017) 025 [arXiv: 1702.02234] [INSPIRE].

[141] S. Descotes-Genon, J. Matias and J. Virto, Understanding the $B \rightarrow K^{*} \mu^{+} \mu^{-}$Anomaly, Phys. Rev. D 88 (2013) 074002 [arXiv:1307.5683] [InSPIRE].

[142] W. Altmannshofer and D.M. Straub, New Physics in $B \rightarrow K^{*} \mu \mu$ ?, Eur. Phys. J. C 73 (2013) 2646 [arXiv: 1308.1501] [INSPIRE].

[143] T. Hurth and F. Mahmoudi, On the LHCb anomaly in $B \rightarrow K^{*} \ell^{+} \ell^{-}$, JHEP 04 (2014) 097 [arXiv: 1312.5267] [INSPIRE].

[144] D. Ghosh, M. Nardecchia and S.A. Renner, Hint of Lepton Flavour Non-Universality in B Meson Decays, JHEP 12 (2014) 131 [arXiv:1408.4097] [INSPIRE].

[145] W. Altmannshofer and D.M. Straub, New physics in $b \rightarrow s$ transitions after LHC run 1, Eur. Phys. J. C 75 (2015) 382 [arXiv:1411.3161] [InSPIRE].

[146] S. Descotes-Genon, L. Hofer, J. Matias and J. Virto, Global analysis of $b \rightarrow$ sll anomalies, JHEP 06 (2016) 092 [arXiv:1510.04239] [INSPIRE].

[147] T. Hurth, F. Mahmoudi and S. Neshatpour, On the anomalies in the latest LHCb data, Nucl. Phys. B 909 (2016) 737 [arXiv:1603.00865] [INSPIRE].

[148] B. Capdevila, S. Descotes-Genon, J. Matias and J. Virto, Assessing lepton-flavour non-universality from $B \rightarrow K^{*} \ell \ell$ angular analyses, JHEP 10 (2016) 075 [arXiv: 1605. 03156] [INSPIRE].

[149] W. Altmannshofer, C. Niehoff, P. Stangl and D.M. Straub, Status of the $B \rightarrow K^{*} \mu^{+} \mu^{-}$ anomaly after Moriond 2017, Eur. Phys. J. C 77 (2017) 377 [arXiv:1703.09189] [INSPIRE].

[150] B. Capdevila, A. Crivellin, S. Descotes-Genon, J. Matias and J. Virto, Patterns of New Physics in $b \rightarrow s \ell^{+} \ell^{-}$transitions in the light of recent data, arXiv:1704.05340 [INSPIRE].

[151] W. Altmannshofer, P. Stangl and D.M. Straub, Interpreting Hints for Lepton Flavor Universality Violation, arXiv: 1704.05435 [INSPIRE]. 
[152] L.-S. Geng, B. Grinstein, S. Jäger, J. Martin Camalich, X.-L. Ren and R.-X. Shi, Towards the discovery of new physics with lepton-universality ratios of $b \rightarrow$ sll decays, arXiv: 1704.05446 [INSPIRE].

[153] M. Ciuchini, A.M. Coutinho, M. Fedele, E. Franco, A. Paul, L. Silvestrini et al., On Flavourful Easter eggs for New Physics hunger and Lepton Flavour Universality violation, arXiv: 1704.05447 [INSPIRE].

[154] C.-J. Lee and J. Tandean, Minimal lepton flavor violation implications of the $b \rightarrow s$ anomalies, JHEP 08 (2015) 123 [arXiv: 1505.04692] [INSPIRE].

[155] T. Hurth, G. Isidori, J.F. Kamenik and F. Mescia, Constraints on New Physics in MFV models: A model-independent analysis of $\Delta F=1$ processes, Nucl. Phys. B 808 (2009) 326 [arXiv:0807.5039] [INSPIRE].

[156] Z. Lalak, S. Pokorski and G.G. Ross, Beyond MFV in family symmetry theories of fermion masses, JHEP 08 (2010) 129 [arXiv:1006.2375] [INSPIRE].

[157] M. Redi and A. Weiler, Flavor and CP Invariant Composite Higgs Models, JHEP 11 (2011) 108 [arXiv: 1106.6357] [INSPIRE].

[158] T. Hurth and F. Mahmoudi, The Minimal Flavour Violation benchmark in view of the latest LHCb data, Nucl. Phys. B $\mathbf{8 6 5}$ (2012) 461 [arXiv:1207.0688] [INSPIRE].

[159] L. Calibbi, P. Paradisi and R. Ziegler, Gauge Mediation beyond Minimal Flavor Violation, JHEP 06 (2013) 052 [arXiv: 1304.1453] [INSPIRE].

[160] F. Bishara, A. Greljo, J.F. Kamenik, E. Stamou and J. Zupan, Dark Matter and Gauged Flavor Symmetries, JHEP 12 (2015) 130 [arXiv:1505.03862] [INSPIRE].

[161] M. Redi, Leptons in Composite MFV, JHEP 09 (2013) 060 [arXiv:1306.1525] [InSPIRE].

[162] X.-G. He, C.-J. Lee, J. Tandean and Y.-J. Zheng, Seesaw Models with Minimal Flavor Violation, Phys. Rev. D 91 (2015) 076008 [arXiv:1411.6612] [INSPIRE].

[163] F. Feruglio, P. Paradisi and A. Pattori, Lepton Flavour Violation in Composite Higgs Models, Eur. Phys. J. C 75 (2015) 579 [arXiv:1509.03241] [inSPIRE].

[164] T. Feldmann, C. Luhn and P. Moch, Lepton-flavour violation in a Pati-Salam model with gauged flavour symmetry, JHEP 11 (2016) 078 [arXiv: 1608.04124] [INSPIRE].

[165] R. Alonso, Dynamical Yukawa Couplings, Ph.D. Thesis, Madrid (2013) [arXiv:1307.1904] [INSPIRE].

[166] A.L. Kagan, G. Perez, T. Volansky and J. Zupan, General Minimal Flavor Violation, Phys. Rev. D 80 (2009) 076002 [arXiv: 0903.1794] [inSPIRE].

[167] S. Weinberg, Baryon and Lepton Nonconserving Processes, Phys. Rev. Lett. 43 (1979) 1566 [INSPIRE].

[168] S.M. Bilenky, J. Hosek and S.T. Petcov, On Oscillations of Neutrinos with Dirac and Majorana Masses, Phys. Lett. B 94 (1980) 495 [inSPIRE].

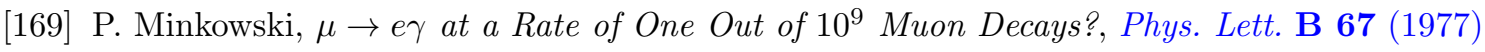
421 [INSPIRE].

[170] M. Gell-Mann, P. Ramond and R. Slansky, Complex Spinors and Unified Theories, Conf. Proc. C 790927 (1979) 315 [arXiv:1306.4669] [inSPIRE].

[171] T. Yanagida, Horizontal Symmetry and Masses of Neutrinos, Prog. Theor. Phys. 64 (1980) 1103 [INSPIRE]. 
[172] R.N. Mohapatra and G. Senjanović, Neutrino Masses and Mixings in Gauge Models with Spontaneous Parity Violation, Phys. Rev. D 23 (1981) 165 [inSPIRE].

[173] J. Schechter and J.W.F. Valle, Neutrino Masses in $\mathrm{SU}(2) \times \mathrm{U}(1)$ Theories, Phys. Rev. D 22 (1980) 2227 [INSPIRE].

[174] E. Bertuzzo, P. Di Bari, F. Feruglio and E. Nardi, Flavor symmetries, leptogenesis and the absolute neutrino mass scale, JHEP 11 (2009) 036 [arXiv:0908.0161] [INSPIRE].

[175] D. Aristizabal Sierra, F. Bazzocchi, I. de Medeiros Varzielas, L. Merlo and S. Morisi, Tri-Bimaximal Lepton Mixing and Leptogenesis, Nucl. Phys. B 827 (2010) 34 [arXiv: 0908.0907] [INSPIRE].

[176] S. Antusch, J. Kersten, M. Lindner and M. Ratz, Running neutrino masses, mixings and CP phases: Analytical results and phenomenological consequences, Nucl. Phys. B 674 (2003) 401 [hep-ph/0305273] [INSPIRE].

[177] S. Antusch, J. Kersten, M. Lindner, M. Ratz and M.A. Schmidt, Running neutrino mass parameters in see-saw scenarios, JHEP 03 (2005) 024 [hep-ph/0501272] [INSPIRE].

[178] J.R. Ellis, A. Hektor, M. Kadastik, K. Kannike and M. Raidal, Running of low-energy neutrino masses, mixing angles and CP-violation, Phys. Lett. B 631 (2005) 32 [hep-ph/0506122] [INSPIRE].

[179] Y. Lin, L. Merlo and A. Paris, Running Effects on Lepton Mixing Angles in Flavour Models with Type I Seesaw, Nucl. Phys. B 835 (2010) 238 [arXiv:0911.3037] [InSPIRE].

[180] S.T. Petcov, The Processes $\mu \rightarrow e+\gamma, \mu \rightarrow e+e+\bar{e}, \nu^{\prime} \rightarrow \nu+\gamma$ in the Weinberg-Salam Model with Neutrino Mixing, Sov. J. Nucl. Phys. 25 (1977) 340 [Erratum ibid. 25 (1977) 698] [INSPIRE].

[181] F. Feruglio, The chiral approach to the electroweak interactions, Int. J. Mod. Phys. A 8 (1993) 4937 [hep-ph/9301281] [InSPIRE].

[182] R. Contino, C. Grojean, M. Moretti, F. Piccinini and R. Rattazzi, Strong Double Higgs Production at the LHC, JHEP 05 (2010) 089 [arXiv: 1002.1011] [INSPIRE].

[183] R. Alonso, M.B. Gavela, L. Merlo, S. Rigolin and J. Yepes, The Effective Chiral Lagrangian for a Light Dynamical "Higgs Particle", Phys. Lett. B 722 (2013) 330 [Erratum ibid. B 726 (2013) 926] [arXiv: 1212.3305] [INSPIRE].

[184] G. Buchalla, O. Catà and C. Krause, Complete Electroweak Chiral Lagrangian with a Light Higgs at NLO, Nucl. Phys. B 880 (2014) 552 [Erratum ibid. B 913 (2016) 475] [arXiv: 1307.5017] [INSPIRE].

[185] I. Brivio, J. Gonzalez-Fraile, M.C. Gonzalez-Garcia and L. Merlo, The complete HEFT Lagrangian after the LHC Run I, Eur. Phys. J. C 76 (2016) 416 [arXiv:1604.06801] [INSPIRE].

[186] LHC Higgs Cross Section Working Group collaboration, D. de Florian et al., Handbook of LHC Higgs Cross sections: 4. Deciphering the Nature of the Higgs Sector, arXiv: 1610.07922 [INSPIRE].

[187] I. Brivio et al., Disentangling a dynamical Higgs, JHEP 03 (2014) 024 [arXiv:1311.1823] [INSPIRE].

[188] I. Brivio, O.J.P. Éboli, M.B. Gavela, M.C. Gonzalez-Garcia, L. Merlo and S. Rigolin, Higgs ultraviolet softening, JHEP 12 (2014) 004 [arXiv:1405.5412] [INSPIRE].

[189] M.B. Gavela, J. Gonzalez-Fraile, M.C. Gonzalez-Garcia, L. Merlo, S. Rigolin and J. Yepes, $C P$ violation with a dynamical Higgs, JHEP 10 (2014) 044 [arXiv:1406.6367] [INSPIRE]. 
[190] R. Alonso, I. Brivio, B. Gavela, L. Merlo and S. Rigolin, Sigma Decomposition, JHEP 12 (2014) 034 [arXiv: 1409.1589] [InSPIRE].

[191] I.M. Hierro, L. Merlo and S. Rigolin, Sigma Decomposition: The CP-Odd Lagrangian, JHEP 04 (2016) 016 [arXiv: 1510.07899] [InSPIRE].

[192] I. Brivio et al., Non-linear Higgs portal to Dark Matter, JHEP 04 (2016) 141 [arXiv: 1511.01099] [INSPIRE].

[193] B.M. Gavela, E.E. Jenkins, A.V. Manohar and L. Merlo, Analysis of General Power Counting Rules in Effective Field Theory, Eur. Phys. J. C 76 (2016) 485 [arXiv: 1601.07551] [INSPIRE].

[194] L. Merlo, S. Saa and M. Sacristán-Barbero, Baryon Non-Invariant Couplings in Higgs Effective Field Theory, Eur. Phys. J. C 77 (2017) 185 [arXiv:1612.04832] [INSPIRE].

[195] I. Brivio et al., ALPs Effective Field Theory and Collider Signatures, arXiv:1701.05379 [INSPIRE].

[196] P. Hernández-Leon and L. Merlo, The Complete Bosonic Basis For A Higgs-Like Dilaton, arXiv: 1703.02064 [INSPIRE].

[197] W. Buchmüller and D. Wyler, Effective Lagrangian Analysis of New Interactions and Flavor Conservation, Nucl. Phys. B 268 (1986) 621 [INSPIRE].

[198] B. Grzadkowski, M. Iskrzynski, M. Misiak and J. Rosiek, Dimension-Six Terms in the Standard Model Lagrangian, JHEP 10 (2010) 085 [arXiv: 1008.4884] [INSPIRE].

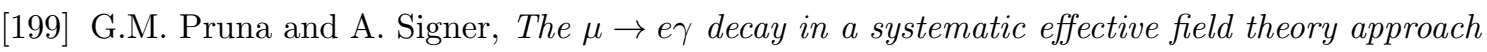
with dimension 6 operators, JHEP 10 (2014) 014 [arXiv:1408.3565] [INSPIRE].

[200] A. Crivellin, S. Davidson, G.M. Pruna and A. Signer, Renormalisation-group improved analysis of $\mu \rightarrow e$ processes in a systematic effective-field-theory approach, JHEP 05 (2017) 117 [arXiv: 1702.03020] [INSPIRE].

[201] R. Kitano, M. Koike and Y. Okada, Detailed calculation of lepton flavor violating muon electron conversion rate for various nuclei, Phys. Rev. D 66 (2002) 096002 [Erratum ibid. D 76 (2007) 059902] [hep-ph/0203110] [INSPIRE].

[202] MEG collaboration, J. Adam et al., New constraint on the existence of the $\mu^{+} \rightarrow e^{+} \gamma$ decay, Phys. Rev. Lett. 110 (2013) 201801 [arXiv:1303.0754] [INSPIRE].

[203] A.M. Baldini et al., MEG Upgrade Proposal, arXiv:1301.7225 [INSPIRE].

[204] BaBAR collaboration, B. Aubert et al., Searches for Lepton Flavor Violation in the Decays $\tau^{ \pm} \rightarrow e^{ \pm} \gamma$ and $\tau^{ \pm} \rightarrow \mu^{ \pm} \gamma$, Phys. Rev. Lett. 104 (2010) 021802 [arXiv:0908.2381] [INSPIRE].

[205] BELle, BELLE-II collaborations, K. Hayasaka, Results and prospects on lepton flavor violation at Belle/Belle II, J. Phys. Conf. Ser. 408 (2013) 012069 [InSPIRE].

[206] SINDRUM II collaboration, W.H. Bertl et al., A Search for muon to electron conversion in muonic gold, Eur. Phys. J. C 47 (2006) 337 [INSPIRE].

[207] Y. Kuno, A search for muon-to-electron conversion at J-PARC: the COMET experiment, Prog. Theor. Exp. Phys. 2013 (2013) $022 \mathrm{C} 01$.

[208] Mu2e collaboration, R.J. Abrams et al., Mu2e Conceptual Design Report, arXiv: 1211.7019 [INSPIRE].

[209] R. Alonso, B. Grinstein and J. Martin Camalich, SU(2) $\times$ U(1) gauge invariance and the shape of new physics in rare B decays, Phys. Rev. Lett. 113 (2014) 241802

[arXiv: 1407.7044] [INSPIRE]. 
[210] G. Hiller and M. Schmaltz, $R_{K}$ and future $b \rightarrow$ sll physics beyond the standard model opportunities, Phys. Rev. D 90 (2014) 054014 [arXiv:1408.1627] [InSPIRE].

[211] R. Alonso, B. Grinstein and J. Martin Camalich, Lepton universality violation and lepton flavor conservation in B-meson decays, JHEP 10 (2015) 184 [arXiv: 1505.05164] [INSPIRE].

[212] F. Feruglio, P. Paradisi and A. Pattori, Revisiting Lepton Flavor Universality in B Decays, Phys. Rev. Lett. 118 (2017) 011801 [arXiv:1606.00524] [INSPIRE].

[213] F. Feruglio, P. Paradisi and A. Pattori, On the Importance of Electroweak Corrections for B Anomalies, arXiv:1705.00929 [INSPIRE].

[214] R. Alonso, P. Cox, C. Han and T.T. Yanagida, Anomaly-free local horizontal symmetry and anomaly-full rare B-decays, arXiv:1704.08158 [INSPIRE].

[215] R. Alonso, P. Cox, C. Han and T.T. Yanagida, Flavoured B - L Local Symmetry and Anomalous Rare B Decays, arXiv:1705.03858 [INSPIRE]. 